\title{
The Synergistic Effect of Ginkgo biloba Extract 50 and Aspirin Against Platelet Aggregation
}

\author{
Jia $\mathrm{Ke}^{\mathrm{l}, *}$ \\ Meng-Ting $\mathrm{Li}^{1}{ }^{1} *$ \\ Ya-jing Huo' \\ Yan-Qiong Cheng ${ }^{2}$ \\ Shu-Fen Guo' \\ Yang $\mathrm{Wu}^{\prime}$ \\ Lei Zhang ${ }^{3}$ \\ Jianpeng $\mathrm{Ma}^{4}$ \\ Ai-Jun $\mathrm{Liu}^{2}$ \\ Yan Han'
}

'Department of Neurology, Yueyang Hospital of Integrated Traditional Chinese and Western Medicine, Shanghai University of Traditional Chinese Medicine, Shanghai, People's Republic of China; ${ }^{2}$ Department of Pharmacology, School of Pharmacy, Second Military Medical University, Shanghai, People's Republic of China; ${ }^{3}$ Department of Vascular Surgery, Yueyang Hospital of Integrated Traditional Chinese and Western Medicine, Shanghai University of Traditional Chinese Medicine, Shanghai, People's Republic of China; ${ }^{4}$ Multiscale Research Institute of Complex Systems, Fudan University, Shanghai, People's Republic of China

*These authors contributed equally to this work

Correspondence: Ai-Jun Liu

Department of Pharmacology, School of

Pharmacy, Second Military Medical

University, 800 Xiangyin Road, Yangpu,

Shanghai, 200433, People's Republic of China

Emailmrliuaijun@I63.com

Yan Han

Department of Neurology, Yueyang Hospital of Integrated Traditional Chinese and Western Medicine, Shanghai University of

Traditional Chinese Medicine, I I0 Ganhe

Road, Hongkou, Shanghai, 200437, People's

Republic of China

Tel +86 2l 5l 322043

Email hanyan.2006@aliyun.com
Purpose: We aimed to investigate potential synergistic antiplatelet effects of Ginkgo biloba extract (GBE50) in combination with aspirin using in vitro models.

Methods: Arachidonic acid (AA), platelet activating factor (PAF), adenosine 5'-diphosphate (ADP) and collagen were used as inducers. The antiplatelet effects of GBE50, aspirin and 1:1 combination of GBE50 and aspirin were detected by microplate method using rabbit platelets. Synergy finder 2.0 was used to analyze the synergistic antiplatelet effect. The compounds in GBE50 were identified by UPLC-Q/TOF-MS analysis and the candidate compounds were screened by TCMSP database. The targets of candidate compounds and aspirin were obtained in TCMSP, CCGs, Swiss target prediction database and drugbank. Targets involving platelet aggregation were obtained from GenCLiP database. Compound-target network was constructed and GO and KEGG enrichment analyses were performed to identify the critical biological processes and signaling pathways. The levels of thromboxane B2 (TXB2), cyclic adenosine monophosphate (cAMP) and PAF receptor (PAFR) were detected by ELISA to determine the effects of GBE50, aspirin and their combination on these pathways.

Results: GBE50 combined with aspirin inhibited platelet aggregation more effectively. The combination displayed synergistic antiplatelet effects in AA-induced platelet aggregation, and additive antiplatelet effects occurred in PAF, ADP and collagen induced platelet aggregation. Seven compounds were identified as candidate compounds in GBE50. Enrichment analyses revealed that GBE50 could interfere with platelet aggregation via cAMP pathway, AA metabolism and calcium signaling pathway, and aspirin could regulate platelet aggregation through AA metabolism and platelet activation. ELISA experiments showed that GBE50 combined with aspirin could increase cAMP levels in resting platelets, and decreased the levels of TXB2 and PAFR.

Conclusion: Our study indicated that GBE50 combined with aspirin could enhance the antiplatelet effects. They exerted both synergistic and additive effects in restraining platelet aggregation. The study highlighted the potential application of GBE50 as a supplementary therapy to treat thrombosis-related diseases.

Keywords: Ginkgo biloba, aspirin, antiplatelet, synergistic effect, Chinese herb, arachidonic acid

\section{Introduction}

When blood vessels are injured, platelets are activated by the exposed subcutaneous matrix and aggregate at the site of injury to stop bleeding. ${ }^{1}$ Thus, platelets play an essential role in physiological hemostasis process, and abnormal platelet aggregation is the pathological basis of thrombosis. Platelet aggregation is caused by the rupture of atherosclerotic plaques or endothelial injury and may lead to acute 
thrombotic occlusive ischemic events when the blood supply to tissues is insufficient or is interrupted by a thrombus, ${ }^{2}$ which is the underlying mechanism for stroke and myocardial infarction as well as the leading cause of morbidity and mortality worldwide. , $^{34}$

Antiplatelet drugs are the main agents used to prevent and treat acute thrombotic occlusive ischemic events. ${ }^{5}$ Platelet aggregation in vivo is mainly induced by endogenous agonists, such as arachidonic acid (AA), adenosine 5'-diphosphate (ADP), platelet activating factor (PAF), collagen and thrombin. ${ }^{6-8}$ These agonists interact with platelet membrane receptors, leading to platelet degranulation, signal transduction and conformational changes of surface glycoproteins, then causing platelet adhesion and aggregation, and many signaling molecules and pathways are involved in these processes. ${ }^{1,8}$ Diverse antiplatelet drugs used in the clinical setting. Some agents target platelet surface receptors, such as the ADP receptor antagonist clopidogrel, while other regulate platelet activation through the inhibition of enzymes involved in intracellular signaling, such as the cyclooxygenase (COX)-1 inhibitor aspirin, the phosphodiesterase inhibitors cilostazol, and dipyridamole. ${ }^{5,9}$ Although the clinical efficacy of these drugs is clear, there are still some patients who experience recurrent ischemic events due to insufficient treatment intensity after treatment. ${ }^{5}$ Thus, the search for novel antiplatelet agents with improved efficacy and safety remains urgent for thrombotic treatment.

Herbs have a long history of prescription use in China and have become a source of drug development for many diseases. ${ }^{10-15}$ Aspirin, the most frequently used antiplatelet agent in the clinic, is extracted from willow bark and can irreversibly inhibit the COX-1 enzyme in the AA pathway. ${ }^{16}$ The development of novel antiplatelet therapy from herbs may be a solution to the current concerns facing antiplatelet therapy. Products of Ginkgo biloba extracts (GBE) have been widely used as botanical medicines and dietary supplements and its main active compounds are lactones and flavonoids. ${ }^{17}$ Ginkgolides, including ginkgolide A, B, C and J, are the most wellstudied natural specific PAF antagonist. ${ }^{18-20}$ PAF is both a platelet aggregation inducer and a mediator of inflammation. ${ }^{19,21} \mathrm{GBE}$ is also known for its remarkable antioxidant capacity due to its rich flavonoid content. ${ }^{22,23}$ GBE exerts it therapeutic effects in a wide range of diseases, such as Alzheimer's disease, tardive dyskinesia, glaucoma and asthma. ${ }^{23-26}$ Studies also have shown that GBE may inhibit platelet aggregation induced by AA,
ADP, thrombin, collagen and PAF. ${ }^{27-30}$ In addition, GBE in combination with cilostazol may potentiate the antiplatelet effects of cilostazol with no prolongation of bleeding time or coagulation time in patients. ${ }^{31}$ Metaanalyses have shown that GBE did not increase the risk of bleeding, either alone or in combination with antiplatelet/anticoagulants. ${ }^{32,33}$ These findings suggest that GBE can effectively inhibit platelet aggregation through multiple pathways, and the combination of GBE and antiplatelet drugs may have synergistic effects in restraining platelet aggregation. Aspirin is the most commonly used antiplatelet drug for the prevention and treatment of ischemic stroke. A clinical trial with small samples showed a decreased platelet aggregation in patients in the GBE and aspirin combination group, but not statistically significant. ${ }^{34}$ There is still no clear conclusion about the synergistic antiplatelet effects of GBE and aspirin. The study on the synergistic antiplatelet effects of GBE and aspirin may provide ideas for new antiplatelet regimens.

Ginkgo biloba extract 50 (GBE50), a standardized GBE product that is the equivalent to the standardized German product as EGb761, has been approved for clinically use by the China Food and Drug Administration (approval number: Z20000049). It comprises 44\% flavones and $6 \%$ lactones and has been widely used in clinical practice. GBE50 has been shown to have antioxidant, antiplatelet and neuroprotective properties. ${ }^{29,35,36}$ The aim of this study was to evaluate the synergistic antiplatelet effects of GBE50 and aspirin on platelet aggregation induced by AA, PAF, ADP and collagen, and the potential mechanisms were also investigated.

\section{Materials and Methods Materials}

AA, ADP and aspirin were obtained from Sigma-Aldrich (St Louis, MO, USA). Collagen (type I) from cow calcaneal tendon was obtained from Macklin (Shanghai, China). PAF was obtained from Cayman Chemical (Ann Arbor, MI, USA). Dimethyl sulfoxide (DMSO) was purchased from Sinopharm (Beijing, China). GBE50 powder (44\% w/w flavonoids and $6 \% \mathrm{w} / \mathrm{w}$ lactones) was provided by SPH Xing Ling Sci. \& Tech. Pharmaceutical Co., Ltd. (Shanghai, China). It is standardized solid extract of Ginkgo biloba leaves with a Chinese SFDA ratification no. Z20000049. Aspirin and GBE50 were dissolved in DMSO for the in vitro studies. ADP and PAF were 
dissolved in DMSO and diluted with deionized water before use. AA was dissolved in DMSO and was alkalized with $1 \%$ potassium carbonate in a ratio of $1: 3$ before use. The collagen was dissolved in $20 \%$ acetic acid solution and diluted with deionized water before use. The Rabbit thromboxanes B2 Elisa kit, Rabbit cAMP Elisa kit and Rabbit PAFR Elisa kit were purchased from Blue Gene Biotech (Shanghai, China).

A Waters Xevo G2-XS Q-TOF-ESI Liquid mass spectrometer was purchased from Waters Corporation (Milford, MA, USA). Acetonitrile, methanol, formic acid and ammonium acetate (chromatographic pure, LC-MS grade) were purchased from Fisher Chemical (Pittsburgh, PA, USA). Experimental reference substance was from Shanghai Institute of Materia Medica, Chinese Academy of Sciences (Shanghai, China).

\section{Animals}

Male New Zealand rabbits weighting 2-2.5 $\mathrm{kg}(\mathrm{n}=7$, 12-16 weeks old, license No. SCXK (Hu) 2015-0005) were purchased from Shanghai Jia Gan Biotechnology Co., Ltd. (Shanghai, China). The rabbits were maintained in a standard laboratory animal facility with free access to food and water and were acclimatized to these conditions for at least 1 week before use. All animal experiments were approved by the Committee on ethics of biomedicine of Second Military Medical University and were conducted according to the National Institutes of Health Guidelines for the Care and Use of Laboratory Animals.

\section{Platelet Preparation}

Whole blood was collected from the ear artery of rabbits. A $3.8 \%$ sodium citrate solution $(1: 9 \mathrm{v} / \mathrm{v}$, citrate: blood $)$ was used as the anticoagulant. Platelet-rich plasma (PRP) and platelet-poor plasma (PPP) were prepared by centrifuging whole blood at $100 \mathrm{~g}$ and $1400 \mathrm{~g}$ for $15 \mathrm{~min}$, respectively. To obtain washed platelets, PRP was centrifuged at $1400 \mathrm{~g}$ for 15 min. $^{37,38}$ The precipitates obtained was washed twice with CGS buffer (sodium chloride, D-glucose, sodium citrate) and suspended in modified tyrode's buffer $(143 \mathrm{mM} \mathrm{NaCl}, 5.4 \mathrm{mM} \mathrm{KCl}, 5 \mathrm{mM}$ HEPES, $0.25 \mathrm{mM}$ Na2HPO4, $0.5 \mathrm{mM} \mathrm{MgCl} 2,1.8 \mathrm{mM}$ $\mathrm{CaCl} 2, \mathrm{pH}$ 7.4). The final platelet counts in PRP/washed platelets were adjusted to $2 \times 10^{8}$ cell $/ \mathrm{mL}$ after counting under optical microscope.

\section{Platelet Aggregation in vitro Assay}

Platelet aggregation was performed using the microplate method as previously described. ${ }^{39,40}$ Briefly, PRP in the 96 well microplate was preincubated for $5 \mathrm{~min}$ at $37^{\circ} \mathrm{C}$ with DMSO solution, GBE50 (final concentration: 0.01, 0.03, $0.1,0.3,1,3 \mathrm{mg} / \mathrm{mL}$ ), aspirin (final concentration: 0.01 , $0.03,0.1,0.3,1,3 \mathrm{mg} / \mathrm{mL}$ ) or $1: 1$ combination of GBE50 and aspirin (final concentration: $0.01 \mathrm{mg} / \mathrm{mL}$ GBE50 + $0.01 \mathrm{mg} / \mathrm{mL}$ aspirin, $0.03 \mathrm{mg} / \mathrm{mL}$ GBE50 $+0.03 \mathrm{mg} / \mathrm{mL}$ aspirin, $0.1 \mathrm{mg} / \mathrm{mL}$ GBE50 $+0.1 \mathrm{mg} / \mathrm{mL}$ aspirin, $0.3 \mathrm{mg} /$ $\mathrm{mL}$ GBE50 + $0.3 \mathrm{mg} / \mathrm{mL}$ aspirin, $1 \mathrm{mg} / \mathrm{mL}$ GBE50 + $1 \mathrm{mg} / \mathrm{mL}$ aspirin and $3 \mathrm{mg} / \mathrm{mL}$ GBE $50+3 \mathrm{mg} / \mathrm{mL}$ aspirin) $(100 \mu \mathrm{L}$ per well and DMSO $<0.5 \% \mathrm{v} / \mathrm{v})$, then the absorbance was determined at $570 \mathrm{~nm}$. Platelet aggregation was triggered by adding AA (final concentration: $1.5 \mathrm{mM}$ ), PAF (final concentration: $4 \mu \mathrm{M}$ ), ADP (final concentration: $20 \mu \mathrm{M}$ ) and collagen (final concentration: $100 \mu \mathrm{g} / \mathrm{mL}$ ), then PRP was incubated for $5 \mathrm{~min}$ and vibrated at $1000 \mathrm{rpm}$. Absorbance was determined at $570 \mathrm{~nm}$ after 5 minutes of incubation. The absorbance of PPP was set as the background value, and the absorbance values before and after stimulation were used to calculate the platelet aggregation rate by using formula below. The inhibition rate of the drug on platelet aggregation was calculated using the DMSO group as the baseline.

$$
\begin{aligned}
& \text { Platelet aggregation }(\%) \\
& =\frac{\text { Absorbance }_{\text {after PRP stimulation }}-\text { Absorbance }_{\mathrm{PPP}}}{\text { Absorbance }_{\text {before PRP stimulation }}-\text { Absorbance }_{\mathrm{PPP}}} \times 100 \%
\end{aligned}
$$

\section{Analysis of Antiplatelet Effects of Drug Combination in vitro}

Dose-effect curves for each drug on platelet aggregation under different inducers were drawn and the half maximal inhibitory concentrations $\left(\mathrm{IC}_{50}\right)$ were calculated by using Prism software, version 8 (GraphPad). Choosing the \%inhibition as the phenotypic response, the antiplatelet effect of each drug combination was analyzed using SynergyFinder2.0 (https://synergyfinder.fimm.fi). ${ }^{41}$ Briefly, the mean inhibitory rates of platelet aggregation of GBE50, aspirin, and the drug combinations at different concentrations were input, respectively. According to the software recommendation, the high single agent (HSA) reference model was selected in this study. This model states that the expected combination effect is the maximum of the single drug responses at corresponding concentrations. Finally, the comprehensive synergy score and synergy maps of drug 
combination were obtained automatically by the software using HSA model. The synergy score was used to evaluate the efficacy of the combination. According to user guide in the website, synergy score was evaluated as follows: scores $<-10$ : the interaction between two drugs was likely to be antagonistic; from -10 to 10 : the interaction between two drugs was likely to be additive; and $>10$ : the interaction between two drugs was likely to be synergistic. ${ }^{41}$

\section{UPLC-Q/TOF-MS Analysis}

Ultra-high-performance liquid chromatographyquadrupole time-of-flight tandem mass spectrometry (UPLC-Q-TOF/MS) analysis was used to identify the components of GBE50. Briefly, $0.1 \mathrm{~g}$ GBE50 was added to a solution of $50 \%$ methanol, followed by eddy oscillation for $1 \mathrm{~min}$ and ultrasonic extraction for $15 \mathrm{~min}$. The supernatant was collected and adjusted to a $25 \mathrm{~mL}$ final volume. The chromatographic method was achieved using Waters ACQUITY UPLC BEH C18 column $(2.1 \mathrm{~mm} \times$ $100 \mathrm{~mm}, 1.7 \mu \mathrm{m})$. The mobile phases A and B consisted of $0.2 \mathrm{~mol} / \mathrm{L}$ ammonium acetate solution (containing $0.1 \%$ formic acid) and acetonitrile, respectively. The mobilephase gradient program was as follows: $0 \sim 10 \mathrm{~min}, 88 \%$ $\mathrm{A} \sim 87 \% \mathrm{~A} ; 10 \sim 13 \mathrm{~min}, 87 \% \mathrm{~A} \sim 85 \% \mathrm{~A} ; 13 \sim 20 \mathrm{~min}, 85 \%$ $\mathrm{A} \sim 84 \% \mathrm{~A} ; 20 \sim 30 \mathrm{~min}, 84 \% \mathrm{~A} \sim 79 \% \mathrm{~A} ; 30 \sim 40 \mathrm{~min}, 79 \%$ $\mathrm{A} \sim 77 \mathrm{~A} ; 40 \sim 50 \mathrm{~min}, 77 \% \mathrm{~A} \sim 70 \mathrm{~A} ; 50 \sim 70 \mathrm{~min}, 70 \%$ $\mathrm{A} \sim 25 \% ; 70 \sim 75 \mathrm{~min} 0 \mathrm{~A}$. The flow rate was $0.4 \mathrm{~mL} / \mathrm{min}$. The column temperature was carried out at $45^{\circ} \mathrm{C}$, and the injection volume was $1 \mu \mathrm{L}$. The mass range was collected by the negative ion detection mode of the electrospray ion source (ESI) with source temperature, $120^{\circ} \mathrm{C}$; cone gas flow, $50 \mathrm{~L} / \mathrm{h}$; desolvation temperature, $500^{\circ} \mathrm{C}$; desolvation gas flow, $800 \mathrm{~L} / \mathrm{h}$; sampling cone, $30 \mathrm{~V}$; extraction cone, 4 $\mathrm{V}$; capillary voltage, $2.5 \mathrm{kV}$; mass-to-charge ratio, $\mathrm{m} / \mathrm{z}$ $50 \sim 1200 \mathrm{Da}$; and lock mass (2 ng/mL Leu-enkephalin) ; m/z 554.2615 [M-H] (negative-ion mode). The data were analyzed using Masslynx V4.1 software (Waters). GBE50 base peak ion current (BPI) chromatogram was obtained by UPLC-Q-TOF/MS analysis. The precise molecular weight and secondary fragment information of the compounds were compared with reference substances and references to obtain all the composition information.

\section{Active Components and Target Screening} The pharmacokinetic parameters of all components were screened using the Traditional Chinese Medicine Systems Pharmacology Database and Analysis Platform (TCMSP, https://tcmspw.com/tcmsp.php). ${ }^{42}$ Oral bioavailability (OB) was calculated as the percentage of an orally administered dose of unchanged drug that reached the systemic circulation, and TCMSP can predict OB through multiple linear regression (MLR), partial least squares regression (PLS) and support-vector machine regression (SVR)-based in silico models. ${ }^{43}$ The drug likeness (DL) is a qualitative concept used in drug design to estimate the "drug-like" features of a prospective compound, and TCMSP can predict DL based on Tanimoto coefficient. ${ }^{44}$ Compounds with high properties of $\mathrm{OB}$ and DL properties are not drugs but have the potential to be drugs. Therefore, compounds identified by UPLC-Q/TOF-MS analysis were screened in the TCMSP. According to recommendations from TCMSP, compounds with $\mathrm{OB} \geq 30 \%$ and $\mathrm{DL} \geq 0.18$ were identified as candidate active compounds and were used in the follow-up study. ${ }^{43,44}$ The target proteins of the candidate active compounds in GBE50 were obtained from the TCMSP database, Computational Chemical Genomics Screening Center (CCGS) database (https://www.cbligand.org/CCGS/) and Swiss Target Prediction database (www.swisstargetprediction.ch). ${ }^{45,46}$ The target proteins of aspirin were obtained from DrugBank (https://go.drugbank.com/). ${ }^{47}$ The GenCLiP database (http://ci.smu.edu.cn/genclip3/analysis.php\#) was used to search targets related to platelet aggregation. ${ }^{48}$ Limiting our analysis to Homo sapiens, and standard names for all protein targets were identified through UniProt (https://www.uniprot.org/).

\section{Construction of the Compounds-Targets Network}

Proteins were uploaded into the bioinformatics platform (http://www.bioinformatics.com.cn/) to create a Venn diagram and to clarify the overlapping relationship between the targets. ${ }^{49}$ Targets were imported into Cytoscape3.8.2 software (https://cytoscape.org/) to establish an active compounds-targets network. ${ }^{50}$

\section{GO and KEGG Pathway Enrichment \\ Analyses}

To further explore the potential synergistic antiplatelet activity of GBE50 and aspirin, we used enrichment analysis. For 108 targets unique to GBE50 and 10 targets unique to aspirin related to platelet aggregation, imported the standard protein names into Metascape database, respectively. For the overlapping targets of 
GBE50 and aspirin on platelet aggregation, imported the standard protein names into ClueGo, a plug-in in Cytoscape. The species was limited to Homo sapiens and the gene ontology (GO) functions and Kyoto Encyclopedia of Genes and Genomes (KEGG) pathway enrichment were then performed, respectively. The GO functions enrichment analysis included cellular component (CC), molecular function (MF), and biological process (BP) analyses. Significantly enriched pathways contained at least 3 genes, and have $\mathrm{P}<0.05 .^{51,52}$

\section{Measurement of TXA2 Generation}

Thromboxane B2 (TXB2) is a stable metabolite of TXA2. Levels of TXB2 were measured to indirectly indicate the formation of TXA2 using the Rabbit thromboxanes B2 Elisa kit. Briefly, PRP were pretreated with GBE50, aspirin, 1:1 mixture of these two drugs and DMSO for 5 $\min$ at $37^{\circ} \mathrm{C}$ before the addition of AA. The reaction was stopped after $5 \mathrm{~min}$ of stimulation by the addition of icecold EDTA solution (16 mM). The levels of TXB2 were measured immediately after sample preparation according to the manufacturer's instructions. According to the results of platelet aggregation assays, GBE50, aspirin and the combination of two drugs almost all showed a good inhibition rate of platelet aggregation at $0.3 \mathrm{mg} / \mathrm{mL}$ concentration, so the $0.3 \mathrm{mg} / \mathrm{mL}$ concentration was used in the measurements of cAMP, TXB2 and PAFR.

\section{Measurement of cAMP and PAFR Levels}

The levels of Cyclic adenosine monophosphate (cAMP) and PAF receptor (PAFR) in platelets were estimated using the Rabbit cAMP Elisa kit and Rabbit PAFR Elisa kit, respectively. The washed platelets were preincubated with GBE50, aspirin, 1:1 mixture of these two drugs and DMSO for $5 \mathrm{~min}$ at $37{ }^{\circ} \mathrm{C}$. Next, platelets were washed three times and resuspended in cool modified tyrode's buffer, and were lysed by repeated freezing and thawing. The supernatant was collected and processed according to the manufacturer's instructions.

\section{Statistical Analysis}

Statistical analyses of the data were carried out using Prism 8 software (GraphPad). Significance was determined by one-way ANOVA and Dunnett's posttest for multiple comparisons. Before Statistical analysis, normality and homogeneity of variance were tested. Nonparametric test was used for data that did not meet the standard. A P-value $<0.05$ was considered statistically significant. Unless stated otherwise, values were expressed as mean \pm SEM. Each experiment was repeated independently at least three times.

\section{Results \\ Concentration Screening of Inducers of Platelet Aggregation}

Platelet aggregation is affected by a number of factors, including the type and the concentration of its inducers. Among these, AA, ADP and collagen are the most used inducers in antiplatelet studies. Ginkgolide, the main component of GBE50, is a well-known natural strong PAFR antagonist. PAF is not only a proinflammatory mediator but also an inducer of platelet aggregation. Therefore, AA (from $0 \mathrm{mM}$ to $1.5 \mathrm{mM}$ ), PAF (from 0 $\mu \mathrm{M}$ to $20 \mu \mathrm{M}$ ), $\mathrm{ADP}$ (from $0 \mu \mathrm{M}$ to $40 \mu \mathrm{M}$ ) and collagen (from $0 \mu \mathrm{g} / \mathrm{mL}$ to $200 \mu \mathrm{g} / \mathrm{mL}$ ) were used to induce platelet aggregation. As shown in Figure $1 \mathrm{~A}-\mathrm{C}$, the "concentration 0 " indicated that the same volume of DMSO instead of inducer was added into the PRP, and for this condition, the platelet aggregation rate was $5.99 \% \pm$ $2.16 \%$. With the addition of different concentrations of inducers, AA, PAF and ADP-induced platelet aggregation in a concentration-dependent manner. In this study, the concentration which induced $60 \%$ to $70 \%$ platelet aggregation was selected to test the inhibitory effects of drugs on platelet aggregation in the follow-up experiments. In Figure 1A-C, the platelet aggregation rates achieved $66.12 \% \pm 1.91 \%, 65.68 \% \pm 4.18 \%$, and $71.71 \% \pm$ $2.59 \%$ using concentration of AA $1.5 \mathrm{mM}$, PAF $4 \mu \mathrm{M}$ and ADP $20 \mu \mathrm{M}$, respectively. Therefore, in subsequent experiments, the induced concentrations of AA, PAF and ADP were $1.5 \mathrm{mM}, 4 \mu \mathrm{M}$ and $20 \mu \mathrm{M}$, respectively. As shown in Figure 1D, within the screening concentration range, the rate of platelet aggregation induced by collagen was relatively low $(44.17 \% \pm 1.17 \%)$. Therefore, the concentration of $100 \mu \mathrm{g} / \mathrm{mL}$ inducing the highest platelet aggregation rate was selected for subsequent experiments.

\section{Effects of GBE50 Alone and in Combination with Aspirin on Platelet Aggregation}

The antiplatelet effects of GBE50 were observed under the stimulation of the above-mentioned inducers. As shown in Figure 2A-D, GBE50 (0.01, 0.03, 0.1, 0.3, 1, $3 \mathrm{mg} / \mathrm{mL})$ alone inhibited platelet aggregation induced by various 
A
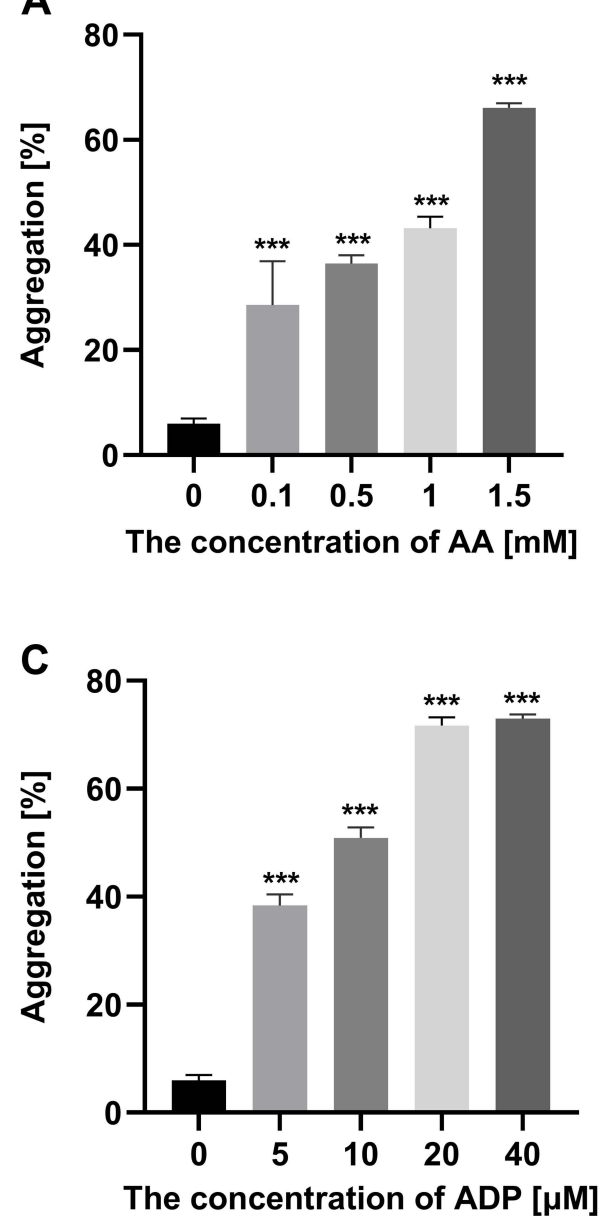

B

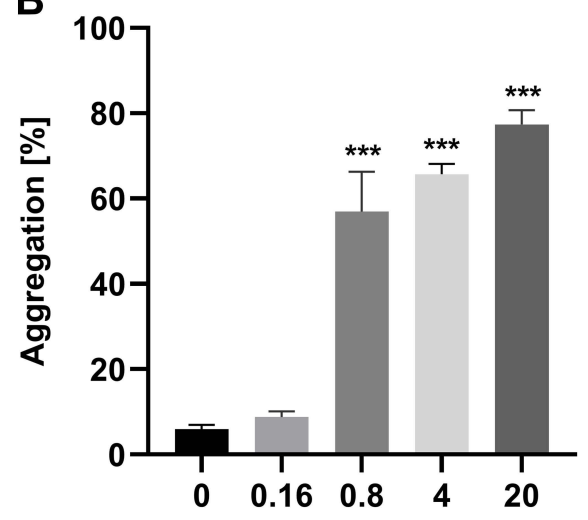

The concentration of PAF $[\mu \mathrm{M}]$

D

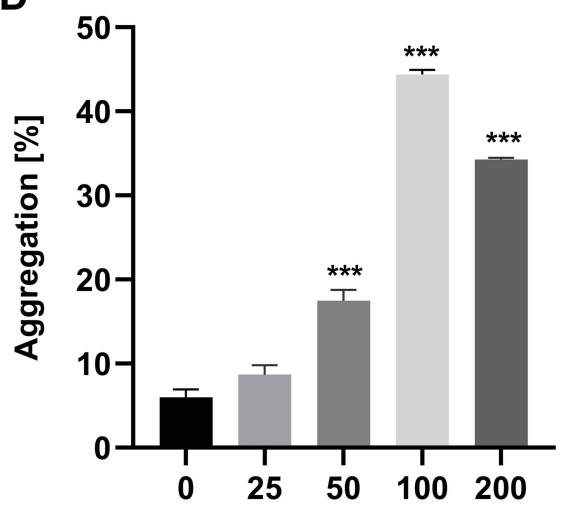

The concentration of collagen $[\mu \mathrm{g} / \mathrm{ml}]$

Figure I The effects of inducers on platelet aggregation in vitro.

Notes: Platelet aggregation induced by (A) AA (from $0 \mathrm{mM}$ to $1.5 \mathrm{mM}$ ), (B) PAF (from $0 \mu \mathrm{M}$ to $20 \mu \mathrm{M}$ ), (C) ADP (from $0 \mu \mathrm{M}$ to $40 \mu \mathrm{M}$ ) and (D) collagen (from $0 \mu \mathrm{g} / \mathrm{mL}$ to $200 \mu \mathrm{g} / \mathrm{mL}$ ) were detected by microplate method. All the data presented as aggregation rate (mean \pm S.E.M.) of three independent experiments, $* * * p<0.00 \mathrm{I}$ compare with concentration 0 .

Abbreviations: AA, arachidonic acid; PAF, platelet-activating factor; ADP, adenosine 5'-diphosphate.

inducers in a concentration-dependent manner, and it showed different degrees of inhibition effects at the same concentration. The dose-effect curves showed that GBE50 had strong inhibitory effects on platelet aggregation induced by collagen and PAF with IC50 values of $0.296 \mathrm{mg} / \mathrm{mL}$ and $0.47 \mathrm{mg} / \mathrm{mL}$, respectively. In contrast, the inhibitory effects of GBE50 on platelet aggregation induced by AA and ADP were relatively weak, and the IC50 values were $3.502 \mathrm{mg} / \mathrm{mL}$ and $6.06 \mathrm{mg} / \mathrm{mL}$, respectively.

To observe the antiplatelet effects of GBE50 combined with aspirin, we tested the inhibitory effects of aspirin alone and GBE50 in combination with aspirin on platelet aggregation exposed to the same inducers. As shown in Figure 2E, aspirin $(0.01,0.03,0.1,0.3,1,3 \mathrm{mg} / \mathrm{mL})$ alone could inhibit platelet aggregation induced by AA, PAF,
ADP and collagen in a concentration-dependent manner, and the IC50 values were $0.234,3.282,1.564$ and $79.78 \mathrm{mg} / \mathrm{mL}$, respectively.

The clinical dosages of GBE50 and aspirin were $120 \mathrm{mg} / \mathrm{d}$ and $100 \mathrm{mg} / \mathrm{d}$, respectively, which was close to 1:1. Therefore, the doses of GBE50 combined with aspirin used in this study were $0.01 \mathrm{mg} / \mathrm{mL}$ GBE50 $+0.01 \mathrm{mg} / \mathrm{mL}$ aspirin, $0.03 \mathrm{mg} / \mathrm{mL}$ GBE50 $+0.03 \mathrm{mg} / \mathrm{mL}$ aspirin, $0.1 \mathrm{mg} / \mathrm{mL}$ GBE50 $+0.1 \mathrm{mg} / \mathrm{mL}$ aspirin, $0.3 \mathrm{mg} / \mathrm{mL}$ GBE50 $+0.3 \mathrm{mg} / \mathrm{mL}$ aspirin, $1 \mathrm{mg} / \mathrm{mlGBE} 50+1 \mathrm{mg} /$ $\mathrm{mL}$ aspirin, $3 \mathrm{mg} / \mathrm{mL}$ GBE50 $+3 \mathrm{mg} / \mathrm{mL}$ aspirin. As shown in Figure 2, the combination of GBE50 and aspirin showed significant antiplatelet effects on AA, PAF, ADP and collagen induced platelet aggregation and the IC50 values were $0.067 \mathrm{mg} / \mathrm{mL}, 0.289 \mathrm{mg} / \mathrm{mL}, 1.474 \mathrm{mg} / \mathrm{mL}$ and $0.149 \mathrm{mg} / \mathrm{mL}$, respectively, and thus, the combination 
A Inducer: AA

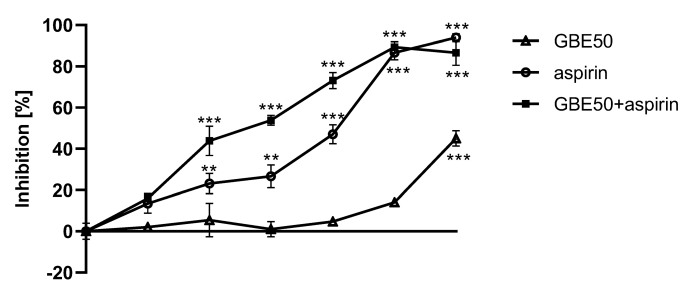

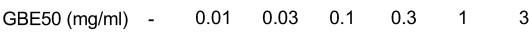

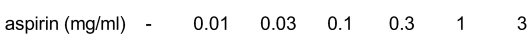

GBE50+aspirin (mg/ml) - $\quad \begin{array}{lllllll}0.01 & 0.03 & 0.1 & 0.3 & 1 & 3\end{array}$

C Inducer: ADP

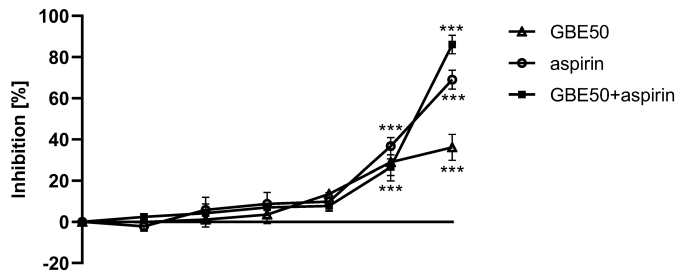

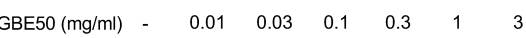

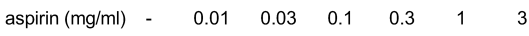

GBE50+aspirin (mg/ml) $\quad-\quad \begin{array}{lllllll}0.01 & 0.03 & 0.1 & 0.3 & 1 & 3\end{array}$
B

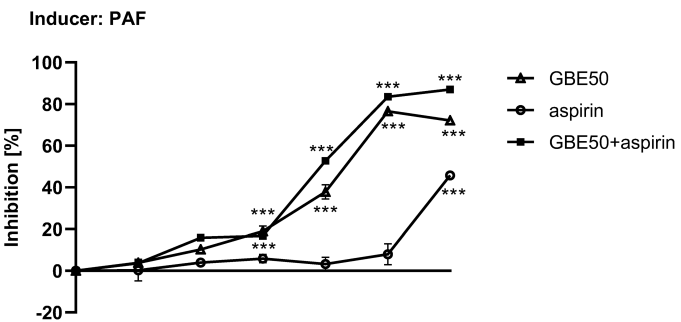

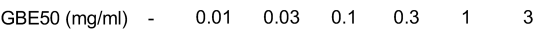

$\begin{array}{lllllllll}\text { aspirin }(\mathrm{mg} / \mathrm{ml}) & - & 0.01 & 0.03 & 0.1 & 0.3 & 1 & 3\end{array}$

GBE50+aspirin (mg/ml) $\quad-\quad \begin{array}{llllll}0.01 & 0.03 & 0.1 & 0.3 & 1 & 3\end{array}$

D Inducer: collagen

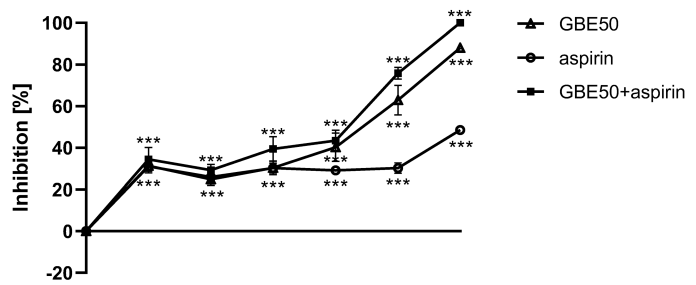

GBE50 (mg/ml) - $\quad 0.01 \quad 0.03 \quad 0.1 \quad 0.3 \quad 1 \quad 3$

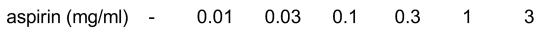

GBE50+aspirin (mg/ml) $\quad-\quad \begin{array}{llllll}0.01 & 0.03 & 0.1 & 0.3 & 1 & 3\end{array}$
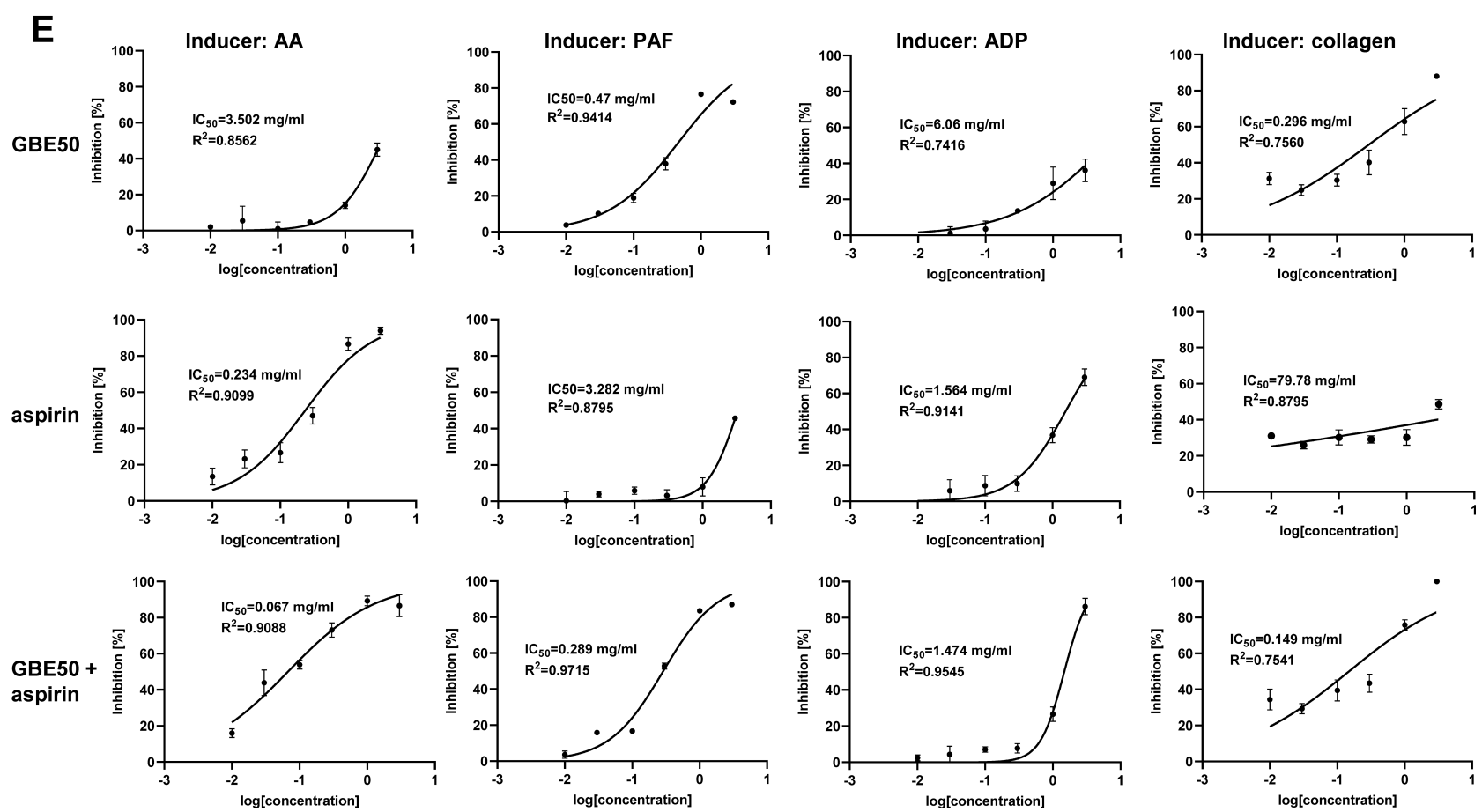

Figure 2 Inhibition of GBE50, aspirin and their combination on platelet aggregation in vitro.

Notes: GBE50, aspirin and their combination concentration-dependently inhibited platelet aggregation in rabbit PRP induced by AA (A), PAF (B), ADP (C), collagen (D). The dose-response curves of drug on inhibition of platelet aggregation under different inducers (E). Results were mean \pm S.E.M. of three independent experiments, $* *$ $<$ 0.01 , *** $\mathrm{p}<0.001$ in comparison to vehicle control.

Abbreviations: GBE50, Ginkgo biloba extract 50; AA, arachidonic acid; PAF, platelet-activating factor; ADP, adenosine 5'-diphosphate; IC 50 , the half maximal inhibitory concentration. 
exhibited superior antiplatelet effects than that of GBE50 or aspirin alone.

\section{Analysis of Synergistic Antiplatelet Effects}

Based on the deviations between observed and expected responses, drug combination can be divided into synergistic (the combination effect is higher than expected) or antagonistic effects (the combination effect is lower than expected) and additive effect synergistic (the combination effect is equal to the expected effect). Our data showed that GBE50 combined with aspirin had stronger antiplatelet effect than the two drugs alone, but the types of interaction between GBE50 and aspirin were unknown. Therefore, we used Synergyfinder 2.0 to further analyze the joint effects of both two drugs. The comprehensive synergy score and synergy maps of drug combination are shown in Figure 3.

In Figure $3 \mathrm{~A}$, under the condition of the AA inducer, the comprehensive synergy score of GBE50 combined with aspirin was 17.506, which indicated that GBE50 and aspirin exhibited synergistic antiplatelet effect in the study dose range. When the combined dose was $0.1 \mathrm{mg} / \mathrm{mL} \mathrm{GBE50+}$ $0.1 \mathrm{mg} / \mathrm{mL}$ aspirin, the synergy score was 30.09 , suggesting that the combined drugs could exert an optimum synergistic antiplatelet effect. In the presence of PAF, the comprehensive synergy score of GBE50 combined with aspirin was 5.349, suggesting that GBE50 and aspirin had additive antiplatelet effects within the range of study dose concentrations. Among them, the dose combination with the most
A

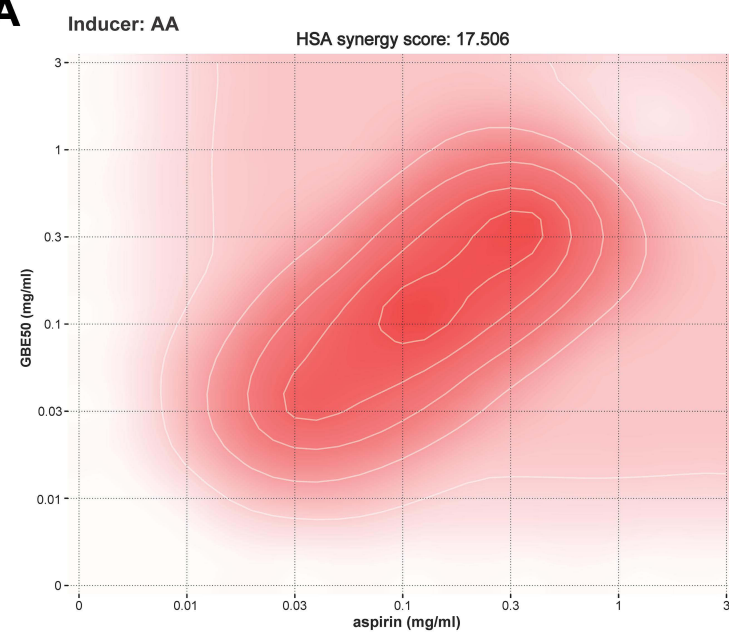

C

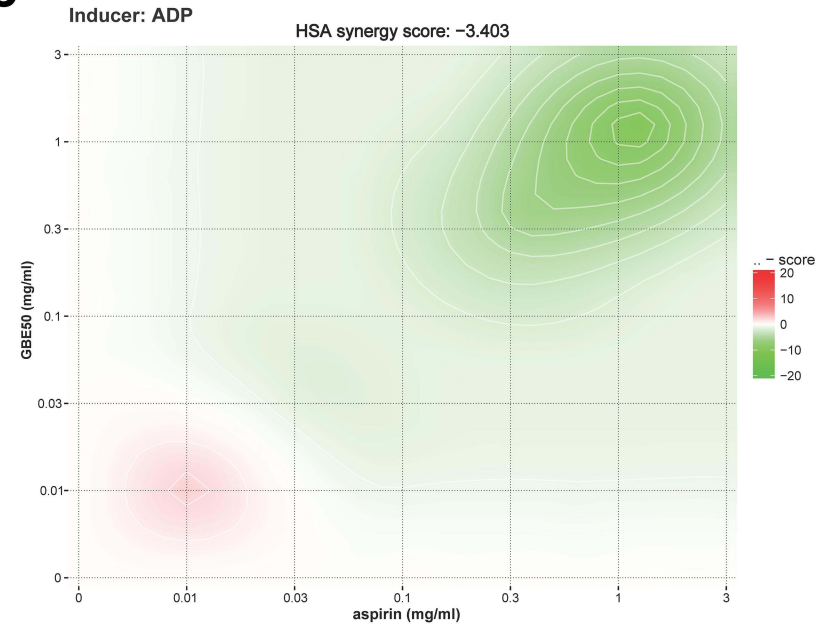

B

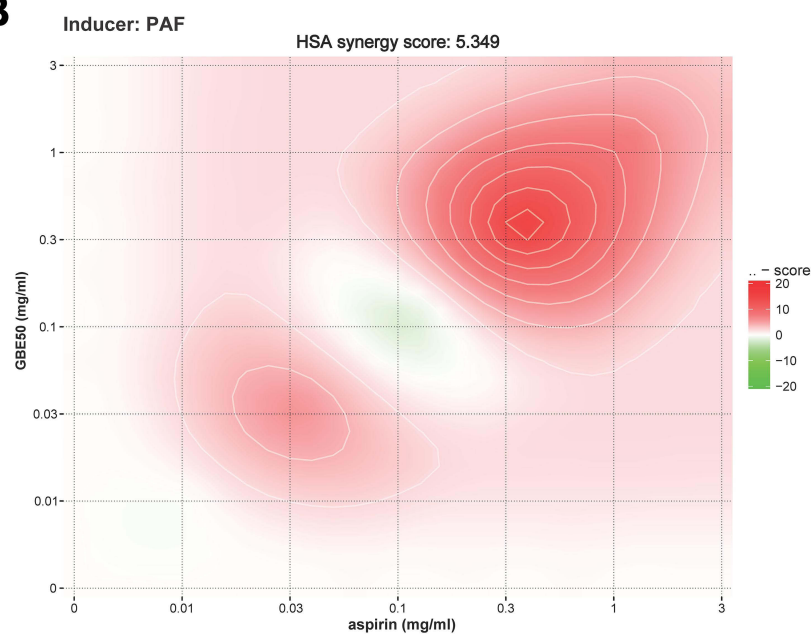

D

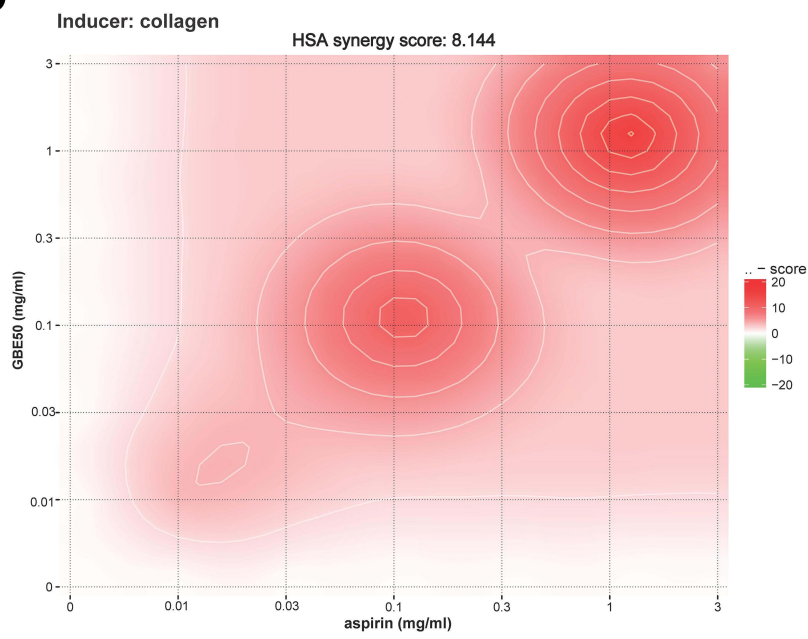

Figure 3 Synergy maps of drug combination.

Notes: The interactive analysis of drug combination in AA (A), PAF (B), ADP (C) and collagen (D) induced platelet aggregation. Drug combinations were $0.0 \mathrm{I} \mathrm{mg} / \mathrm{mL}$

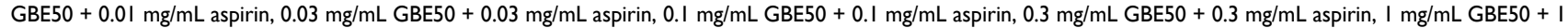
$\mathrm{mg} / \mathrm{mL}$ aspirin and $3 \mathrm{mg} / \mathrm{mL}$ GBE50 $+3 \mathrm{mg} / \mathrm{mL}$ aspirin. Results were mean \pm S.E.M. of three independent experiments. In the figure, the synergetic and antagonistic dose areas were highlighted in red and green.

Abbreviations: GBE50, Ginkgo biloba extract 50; AA, arachidonic acid; PAF, platelet-activating factor; ADP, adenosine 5'-diphosphate; HSA, high single agent. 
synergistic effect of the combined drugs was $0.3 \mathrm{mg} / \mathrm{mL}+$ $0.3 \mathrm{mg} / \mathrm{mL}$, and the synergy score was 15.7 . Under the condition of ADP-induced platelet aggregation, the comprehensive synergy score of drug combination was - 3.403, which indicated that GBE50 and aspirin exhibited additive antiplatelet effects within the study dose range. However, there was no optimum synergistic antiplatelet effect detected within the study dose range. In the collagen-induced platelet aggregation, the comprehensive synergy score was 8.144, suggesting that GBE50 and aspirin had additive antiplatelet effects within the study dose range. Among the platelet inducers tested, the dose combination with the most synergistic effect of the combination GBE50 and aspirin was $1 \mathrm{mg} / \mathrm{mL}+1 \mathrm{mg} / \mathrm{mL}$, and the synergy score was 16.69 .

\section{Identification of the Responsible Chemical Constituent of GBE50}

The BPI chromatogram of GBE50 obtained by UPLC-Q/ TOF-MS is shown in Figure 4. Following comparison with literature and reference substance, 73 compounds were identified, including terpene lactones, flavonoids and their glycosides, organic acids, flavanols and diflavones. Among them, 39 components were compared with reference substances and the relevant information was searched in databases such as PubChem and TCMSP. These 73 chemical constituents accounted for about $75.6 \%$ of the
GBE50 preparation, which embodies the traditional Chinese medicine of multicomponent, multiple targets, the characteristics of wholeness. The related information for each component for shown in Table 1.

\section{Network Analysis}

In this study, with $\mathrm{OB} \geq 30 \%$ and $\mathrm{DL} \geq 0.18$ as the selection criteria, 10 components were selected among the 39 compounds identified by UPLC-Q/TOF-MS, 3 components failed to be associated with relevant targets were removed from the analysis. Finally, seven components were identified as candidate active components. They were ginkgolide B, isorhamnetin, kaempferol, catechin, luteolin, laricitrin and quercetin. Detailed information regarding these molecules was summarized in Table 2.

Based on the TCMSP, CCGS, Swiss Target Prediction and DrugBank databases, 274 targets of seven GBE50 active compounds and 33 targets of aspirin were obtained. A total of 1004 targets of platelet aggregation were collected from the GenCLiP database. As shown in Figure 5A, GBE50 and aspirin could influence platelet aggregation through ten common targets including PTGS1, PTGS2 and IKBKB. GBE50 could also act on platelet aggregation through 108 additional targets such as NOS2, ESR1 and PPARG, while aspirin could only act on platelet aggregation through ten additional targets including MAPK3, ITGA2B and EDNRA.

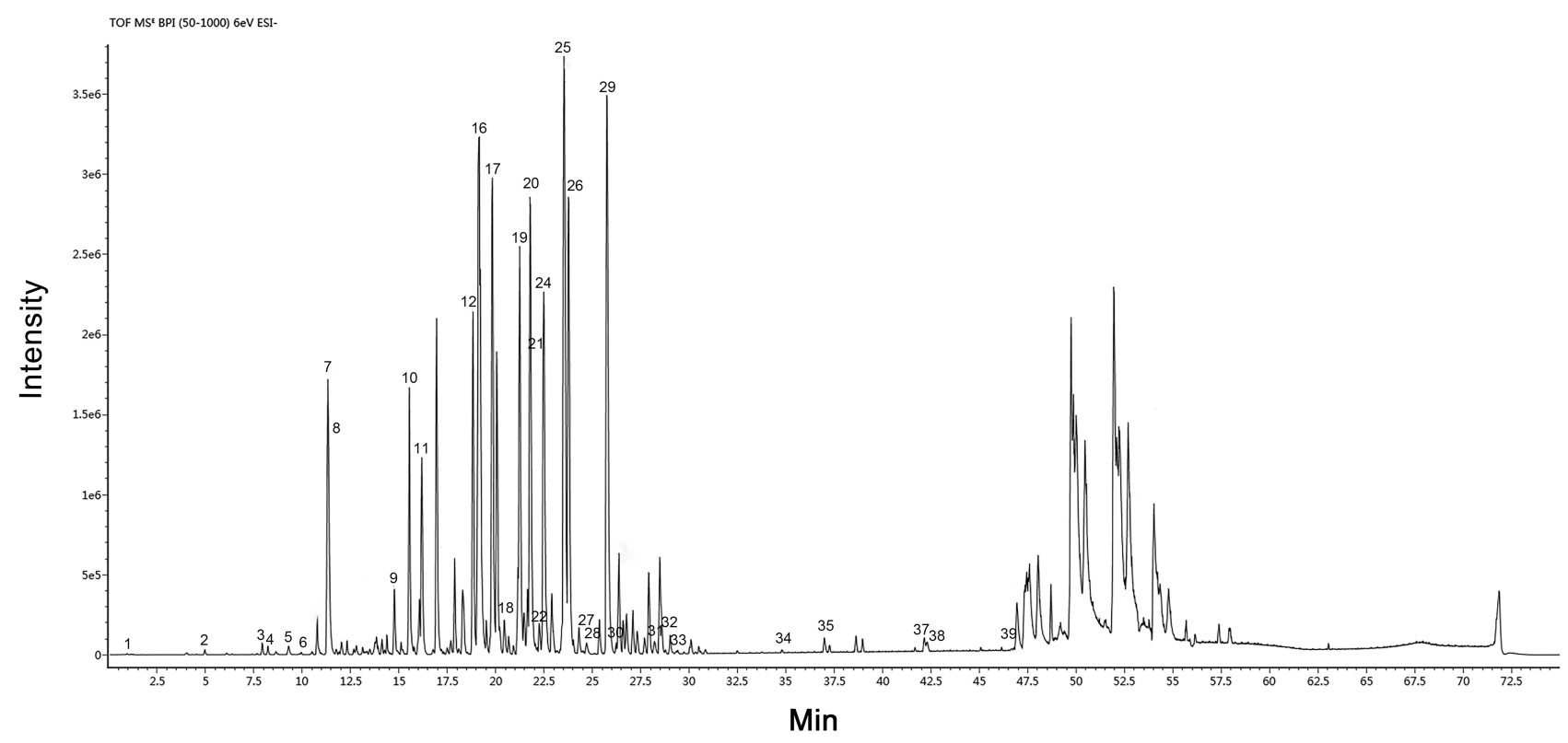

Figure 4 The base peak ion current chromatogram of GBE50.

Note: The constituents represented by the peak number are shown in Table I.

Abbreviations: BPI, base peak ion; Min, minute. 
Table I Constituents Identified in GBE50

\begin{tabular}{|c|c|c|c|c|c|}
\hline No. & CAS Number & Name & Elemental Composition & $m / z[M-H]-$ & $t_{R} /$ Min \\
\hline I & $77-95-2$ & Quinic Acid & $\mathrm{C}_{7} \mathrm{H}_{12} \mathrm{O}_{6}$ & 191.055 & 1.26 \\
\hline 2 & $99-50-3$ & Protocatechuic Acid & $\mathrm{C}_{7} \mathrm{H}_{6} \mathrm{O}_{4}$ & 153.019 & 4.89 \\
\hline 3 & $5127-64-0$ & Gallocatechin Gallate & $\mathrm{C}_{15} \mathrm{H}_{14} \mathrm{O}_{7}$ & 305.067 & 7.87 \\
\hline 4 & $7295-85-4$ & Catechin & $\mathrm{C}_{15} \mathrm{H}_{14} \mathrm{O}_{6}$ & 289.072 & 8.15 \\
\hline 5 & $331-39-5$ & Caffeic Acid & $\mathrm{C}_{9} \mathrm{H}_{8} \mathrm{O}_{4}$ & 179.034 & 9.77 \\
\hline 6 & $99-96-7$ & P-Hydroxybenzoic Acid & $\mathrm{C}_{7} \mathrm{H}_{6} \mathrm{O}_{3}$ & 137.024 & 9.81 \\
\hline 7 & $33570-04-6$ & Bilobalide & $\mathrm{C}_{15} \mathrm{H}_{18} \mathrm{O}_{8}$ & 325.093 & $\mid 1.31$ \\
\hline 8 & $970-74-1$ & (-)-Epigallocatechin & $\mathrm{C}_{15} \mathrm{H}_{14} \mathrm{O}_{7}$ & 289.072 & 11.69 \\
\hline 9 & 107438-79-9 & Ginkgolide J & $\mathrm{C}_{20} \mathrm{H}_{24} \mathrm{O}_{10}$ & 423.129 & 14.77 \\
\hline 10 & $1529 \mid-76-6$ & Ginkgolide C & $\mathrm{C}_{20} \mathrm{H}_{24} \mathrm{O}_{11}$ & 439.124 & 15.55 \\
\hline II & $19833-12-6$ & Myricetin 3-rha-(I-6)-glucoside & $\mathrm{C}_{27} \mathrm{H}_{30} \mathrm{O}_{17}$ & 625.140 & 16.12 \\
\hline 12 & $55804-74-5$ & Kaempferol 3-(2,6-dirha)-glucoside & $\mathrm{C}_{33} \mathrm{H}_{40} \mathrm{O}_{19}$ & 739.207 & 18.80 \\
\hline 13 & $482-35-9$ & Quercetin 3-glucoside & $\mathrm{C}_{21} \mathrm{H}_{20} \mathrm{O}_{12}$ & 463.087 & 19.00 \\
\hline 14 & $153-18-4$ & Rutin & $\mathrm{C}_{27} \mathrm{H}_{30} \mathrm{O}_{16}$ & 609.146 & 19.08 \\
\hline 15 & $|529|-75-5$ & Ginkgolide A & $\mathrm{C}_{20} \mathrm{H}_{24} \mathrm{O}_{9}$ & 407.134 & 19.20 \\
\hline 16 & 104472-68-6 & Isorhamnetin 3-(6-cglc) -(I-2)-rhamnoside & $\mathrm{C}_{34} \mathrm{H}_{42} \mathrm{O}_{20}$ & 769.217 & 19.23 \\
\hline 17 & $1529 \mid-77-7$ & Ginkgolide B & $\mathrm{C}_{20} \mathrm{H}_{24} \mathrm{O}_{10}$ & 423.129 & 19.86 \\
\hline 18 & $39986-90-8$ & Laricitrin 3-rha-(I-6)-glucoside & $\mathrm{C}_{28} \mathrm{H}_{32} \mathrm{O}_{17}$ & 639.156 & 20.03 \\
\hline 19 & $578-74-5$ & Kaempferol 3-rhamnoside & $\mathrm{C}_{21} \mathrm{H}_{20} \mathrm{O}_{10}$ & 431.098 & 21.14 \\
\hline 20 & $480-10-4$ & Kaempferol 3-glucoside & $\mathrm{C}_{21} \mathrm{H}_{20} \mathrm{O}_{11}$ & 447.093 & 21.62 \\
\hline 21 & $17650-84-9$ & Kaempferol 3-glc-(I-2)-rhamnoside & $\mathrm{C}_{27} \mathrm{H}_{30} \mathrm{O}_{15}$ & 593.151 & 21.74 \\
\hline 22 & $504 \mid-82-7$ & Isorhamnetin 3-glucoside & $\mathrm{C}_{22} \mathrm{H}_{22} \mathrm{O}_{12}$ & 477.103 & 22.26 \\
\hline 23 & $604-80-8$ & Isorhamnetin 3-rha-(I-6)-glucoside & $\mathrm{C}_{28} \mathrm{H}_{32} \mathrm{O}_{16}$ & 623.161 & 22.46 \\
\hline 24 & $53430-50-5$ & Syringetin 3-glc-(I-2)-rhamnoside & $\mathrm{C}_{29} \mathrm{H}_{34} \mathrm{O}_{17}$ & 653.171 & 22.88 \\
\hline 25 & $|4306|-65-8$ & Quercetin 3-(6-cglc) -(I-2)-rhamnoside & $\mathrm{C}_{36} \mathrm{H}_{36} \mathrm{O}_{18}$ & 755.180 & 23.52 \\
\hline 26 & $40437-72-7$ & Kaempferol 3-rha-(I-6)-glucoside & $\mathrm{C}_{27} \mathrm{H}_{30} \mathrm{O}_{15}$ & 593.151 & 23.70 \\
\hline 27 & $117-39-5$ & Quercetin & $\mathrm{C}_{15} \mathrm{H}_{10} \mathrm{O}_{7}$ & 301.036 & 24.68 \\
\hline 28 & $53472-37-0$ & Laricitrin & $\mathrm{C}_{16} \mathrm{H}_{12} \mathrm{O}_{8}$ & 331.047 & 24.68 \\
\hline 29 & III957-48-3 & Kaempferol 3-(6-cglc) - (I-2) -rhamnoside & $\mathrm{C}_{36} \mathrm{H}_{36} \mathrm{O}_{17}$ & 739.185 & 25.75 \\
\hline 30 & $491-70-3$ & Luteolin & $\mathrm{C}_{15} \mathrm{H}_{10} \mathrm{O}_{6}$ & 285.673 & 25.94 \\
\hline 31 & $520-18-3$ & Kaempferol & $\mathrm{C}_{15} \mathrm{H}_{10} \mathrm{O}_{6}$ & 285.041 & 28.20 \\
\hline 32 & $520-36-5$ & Apigenin & $\mathrm{C}_{15} \mathrm{H}_{10} \mathrm{O}_{5}$ & 269.046 & 28.73 \\
\hline 33 & $480-19-3$ & Isorhamnetin & $\mathrm{C}_{16} \mathrm{H}_{12} \mathrm{O}_{7}$ & 315.051 & 29.31 \\
\hline 34 & $1617-53-4$ & Amentoflavone & $\mathrm{C}_{30} \mathrm{H}_{18} \mathrm{O}_{10}$ & 537.082 & 34.79 \\
\hline 35 & $521-32-4$ & Bilobetin & $\mathrm{C}_{31} \mathrm{H}_{20} \mathrm{O}_{10}$ & 551.140 & 37.00 \\
\hline 36 & $2|763-7|-3$ & Sequoiaflavone & $\mathrm{C}_{31} \mathrm{H}_{20} \mathrm{O}_{10}$ & 551.099 & 41.15 \\
\hline 37 & $48 I-46-9$ & Ginkgetin & $\mathrm{C}_{32} \mathrm{H}_{22} \mathrm{O}_{10}$ & 565.114 & 42.19 \\
\hline 38 & $548-19-6$ & Isoginkgetin & $\mathrm{C}_{32} \mathrm{H}_{22} \mathrm{O}_{10}$ & 565. II4 & 42.33 \\
\hline 39 & $521-34-6$ & Sciadopitysin & $\mathrm{C}_{33} \mathrm{H}_{24} \mathrm{O}_{10}$ & 579.130 & 46.81 \\
\hline
\end{tabular}

Abbreviation: GBE50, Ginkgo biloba extracts 50.

Next, the compound-target network (Figure 5B) was constructed to uncover the interaction between GBE50 active compounds and aspirin on platelet aggregation. This network contained 136 nodes (128 genes and eight compounds) and 265 edges, the more edges a component or target had, the more important they were to this network. The network showed that quercetin, luteolin, kaempferol and isorhamnetin might be the most important antiplatelet components of GBE50 to exert antiplatelet effects, as they had the most edges. Among the overlapping antiplatelet targets of GBE50 and aspirin, PTGS1 and PTGS2 had the most edges, 
Table 2 The Information of 7 Candidate Active Components in GBE50

\begin{tabular}{|l|l|l|l|l|l|}
\hline Compound Name & Molecule ID & PubChem CID & MW & OB (\%) & DL \\
\hline Ginkgolide B & MOL0II586 & 65243 & 424.44 & 44.38 & 0.73 \\
Isorhamnetin & MOL000354 & 5281654 & 316.28 & 49.6 & 0.31 \\
Kaempferol & MOL000422 & 5280863 & 286.25 & 41.88 & 0.24 \\
Catechin & MOL000492 & 9064 & 290.29 & 54.83 & 0.24 \\
Luteolin & MOL000006 & 5280445 & 286.25 & 36.16 & 0.25 \\
Laricitrin & MOL009278 & 5282154 & 332.28 & 35.38 & 0.34 \\
Quercetin & MOL000098 & 5280343 & 302.25 & 46.43 & 0.28 \\
\hline
\end{tabular}

Abbreviations: GBE50, Ginkgo biloba extracts 50; MW, molecular weight; OB, oral bioavailability; DI, druglikeness.

indicating that they played a key role in the combined antiplatelet effects of GBE50 and aspirin.

\section{KEGG Pathway and GO Function Enrichment Analysis}

GBE50 and aspirin shared common targets as well as individual targets. To explore the possible mechanism of their combined use to enhance antiplatelet effects, KEGG pathway and GO functional enrichment analysis were performed. The 108 additional targets of GBE50 on platelet aggregation were enriched in $167 \mathrm{KEGG}$ pathways, and the top 20 pathways are shown in Figure 6A. Among them, the cAMP signaling pathway, complement and coagulation cascades and calcium signaling pathway were directly related to platelet aggregation. Ten terms were significantly enriched in MF, BP and $\mathrm{CC}$ in the GO analysis (Figure 6B). The results revealed that these 108 targets were strongly associated with molecular functions such as cytokine receptor binding, transcription factor binding and integrin binding, biological processes such as cellular response to lipid, cell migration and cell adhesion, and cellular components such as the extracellular matrix and platelet alpha granule lumen.

Aspirin's ten targets related to platelet aggregation were enriched in hypertrophic cardiomyopathy, platelet activation and oxytocin signaling pathway (Figure 6C), among which platelet activation was directly related to platelet aggregation. GO analysis (Figure 6D) showed that these ten targets might regulate the action of related enzymes by affecting cellular components including platelet alpha granules, thereby affecting biological processes such as platelet degranulation and activation in platelet aggregation.

The overlapping targets of GBE50 and aspirin on platelet aggregation were enriched in 27 KEGG pathways; the top 20 enriched pathways are shown in Figure 6E. Among these, arachidonic acid metabolism was directly related to platelet aggregation, especially AA-induced platelet aggregation. GO functional analysis (Figure 6F) also showed that these ten overlapping targets could regulate the metabolic process of AA. The above results showed that the antiplatelet effect of GBE50 might be related to cAMP signaling pathway and AA metabolism, and AA
A

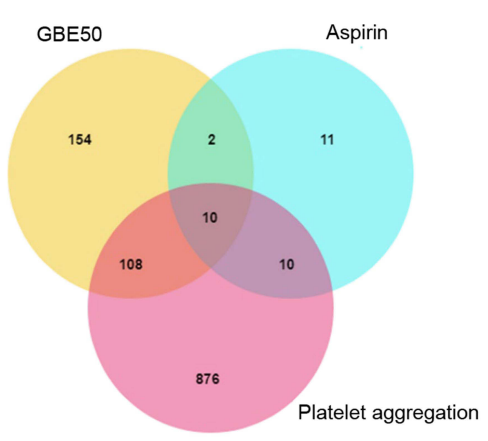

B

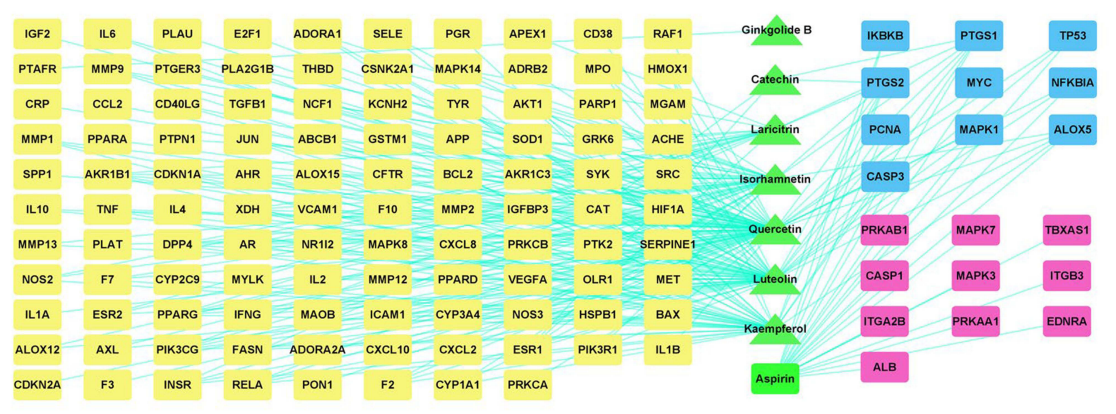

Figure 5 Target prediction and network analysis.

Notes: The Venn diagram of target of GBE50 active compounds, aspirin and platelet aggregation (A). Compound-target visualization network (B). The green rectangle represented aspirin; The green triangle represented the candidate antiplatelet constituents of GBE50; The blue rectangle represented the common targets of GBE50 active components and aspirin on platelet aggregation; The yellow rectangle represented 108 additional targets of GBE50 active components on platelet aggregation; The red rectangle represented 10 additional targets of aspirin on platelet aggregation. The edges between targets and components represented their interactions. 
A

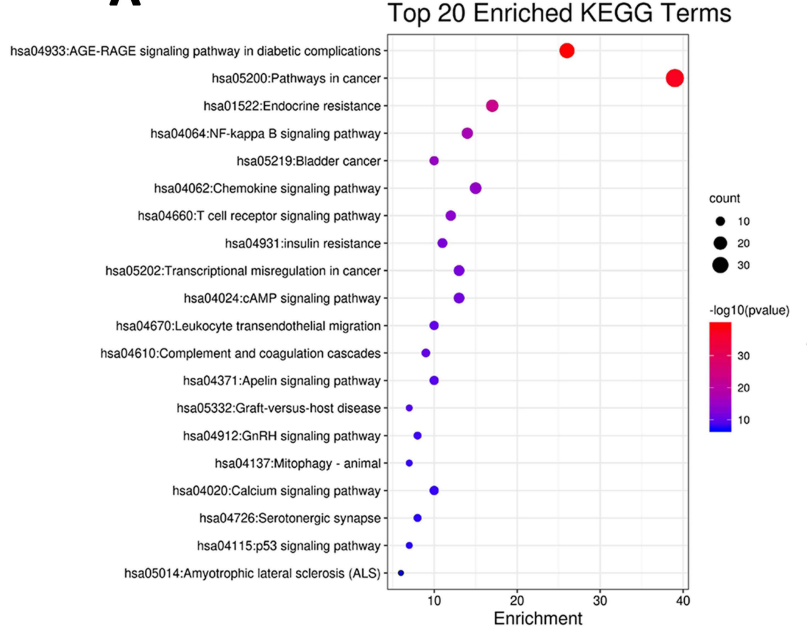

C

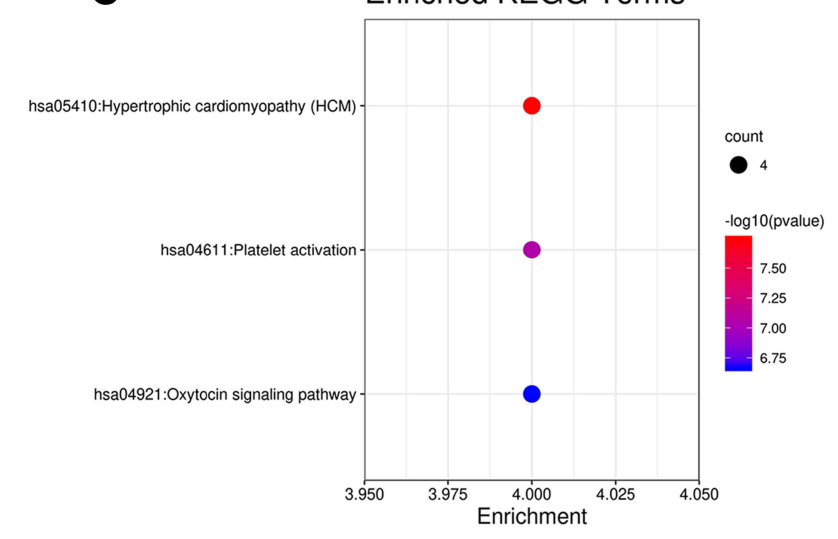

\section{E}

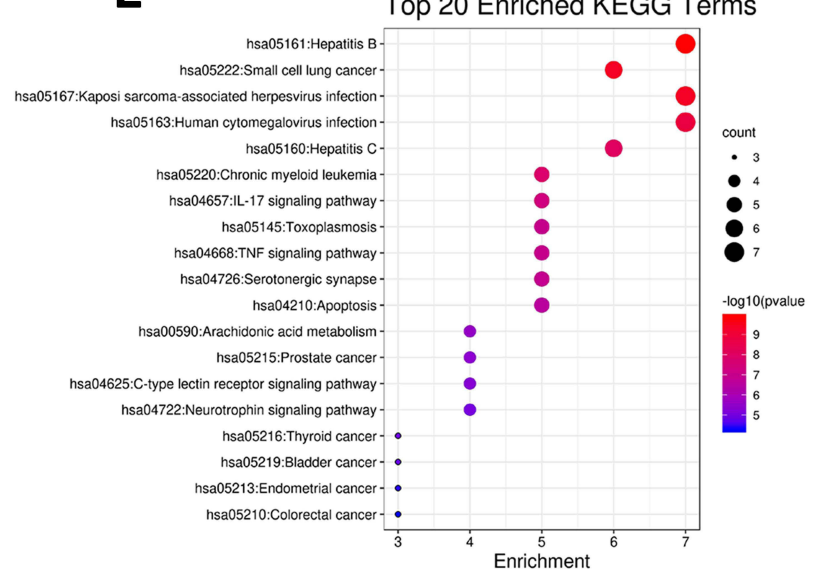

B

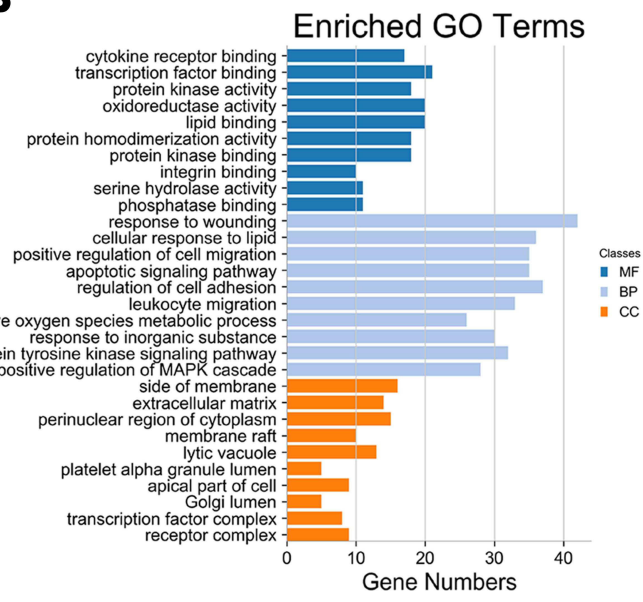

\section{D}

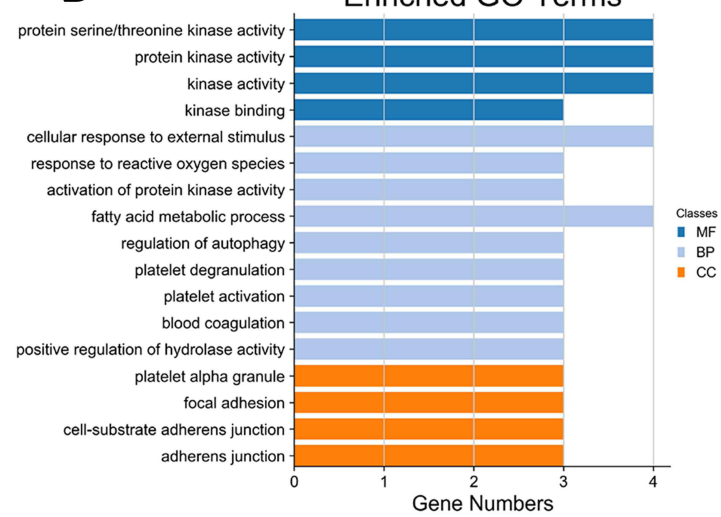

$\mathbf{F}$

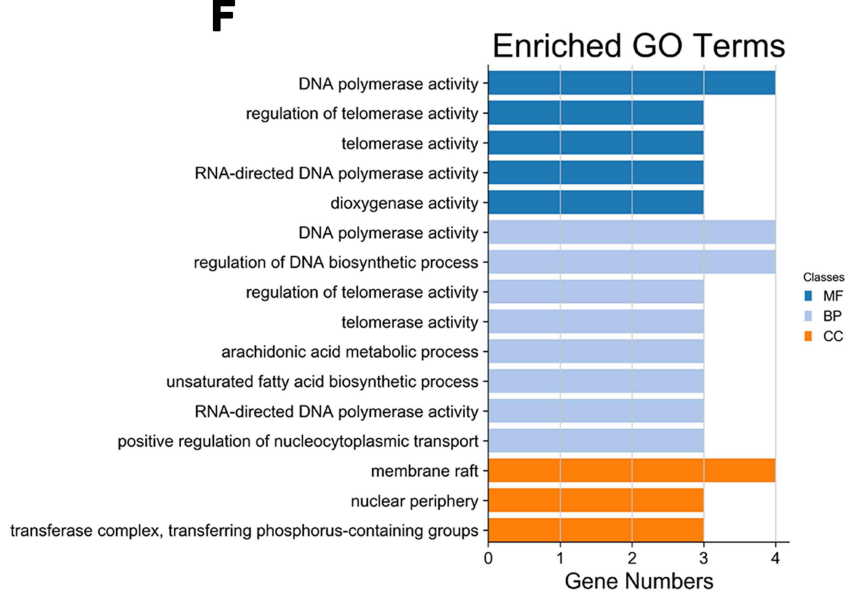

Figure 6 KEGG pathway and GO functional enrichment analysis.

Notes: The KEGG pathway enrichment results of 108 additional targets of GBE50 active compounds (A), 10 additional targets of aspirin (C) and the overlapping 10 targets of GBE50 active compounds and aspirin (E). GO functional analysis of 108 additional targets of GBE50 active compounds (B), 10 additional targets of aspirin (D) and the overlapping 10 targets of GBE50 active compounds and aspirin (F).

Abbreviations: KEGG, Kyoto Encyclopedia of Genes and Genomes; GO, gene ontology; MF, molecular function; BP, biological process; CC, cellular component. 
metabolism signal might be involved in the antiplatelet effect of GBE50 combined with aspirin.

\section{Promotion of cAMP Synthesis by GBE50, Aspirin, and Their Combination in Platelets}

The KEGG pathway enrichment analysis indicated that GBE50 active compounds could influence platelet aggregation by regulating the cAMP signaling pathway. We therefore assessed the effects of GBE50, aspirin and their combination in this signaling pathway in resting platelets. As illustrated in Figures 7A, $0.3 \mathrm{mg} / \mathrm{mL}$ GBE50 and the $0.3 \mathrm{mg} / \mathrm{mL}$ GBE50 $+0.3 \mathrm{mg} / \mathrm{mL}$ aspirin combination promoted the synthesis of cAMP compared with the vehicle group, but aspirin alone exerted no. Compared with GBE50 or aspirin alone, the combination of GBE50 and aspirin significantly increased cAMP levels.

\section{Effects of GBE50, Aspirin, and the Combination of the Two Drugs on TXB2 Secretion}

GBE50 and aspirin exhibited synergistic antiplatelet effect on AA-induced platelet aggregation. The KEGG and GO analysis of the overlapping ten targets also showed that GBE50 and aspirin could influence AA metabolism.
TXA2 is a bioactive metabolite of AA. Therefore, we evaluated the effects of GBE50, aspirin and the combination of the two drugs on the secretion of TXA2 following AA-induced platelet aggregation. TXB2 levels were measured as an indicator of TXA2 formation. Treatment with aspirin $0.3 \mathrm{mg} / \mathrm{mL}$ alone or $0.3 \mathrm{mg} / \mathrm{mL}$ GBE $50+0.3 \mathrm{mg} /$ $\mathrm{mL}$ aspirin significantly inhibited the secretion of TXB2 compared with the vehicle treated group (Figure 7B). Compared with the vehicle group, the GBE50 treated group resulted in lower TXB2 level, but there was no significant difference between the two groups. Overall, $0.3 \mathrm{mg} / \mathrm{mL}$ GBE50 $+0.3 \mathrm{mg} / \mathrm{mL}$ aspirin displayed stronger inhibitory effects than GBE50 or aspirin alone.

\section{Effects of GBE50, Aspirin, and Their Combination on Levels of PAFR}

KEGG pathway enrichment showed that GBE50 could regulate platelet aggregation by acting on the calcium signaling pathway. PAFR protein was significantly enriched into this pathway. Thus, we evaluated the effects of treatment with GBE50, aspirin and their combination on level of PAFR on platelets in PAF-induced platelet aggregation. In Figure $7 \mathrm{C}$, exposure to $0.3 \mathrm{mg} / \mathrm{mL}$ GBE50 or the combination of $0.3 \mathrm{mg} / \mathrm{mL}$ GBE50 $+0.3 \mathrm{mg} / \mathrm{mL}$ aspirin significantly decreased the levels of PAFR on platelet compared treatment with vehicle. Aspirin treatments,
A

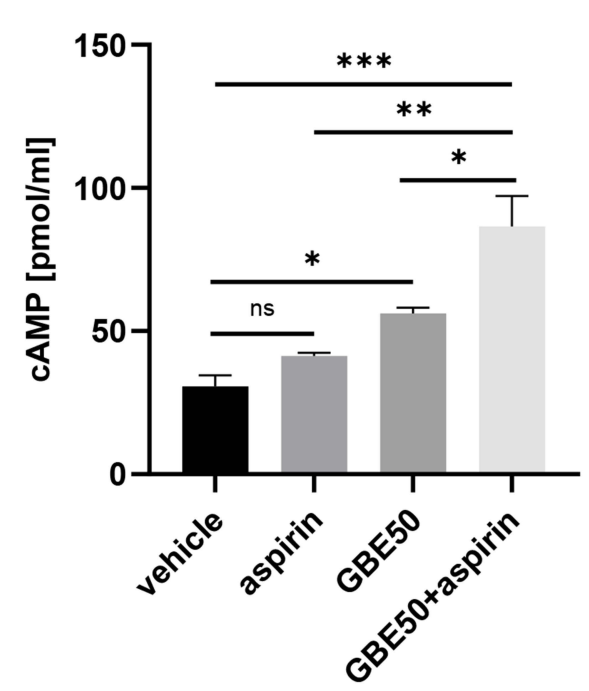

B

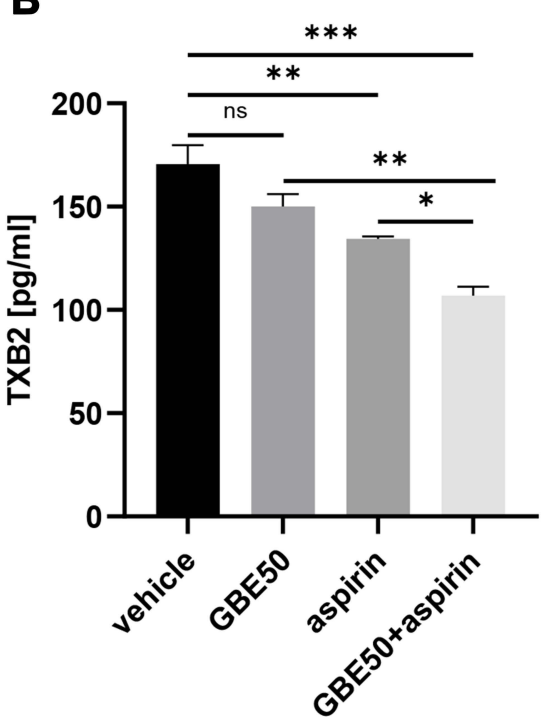

C

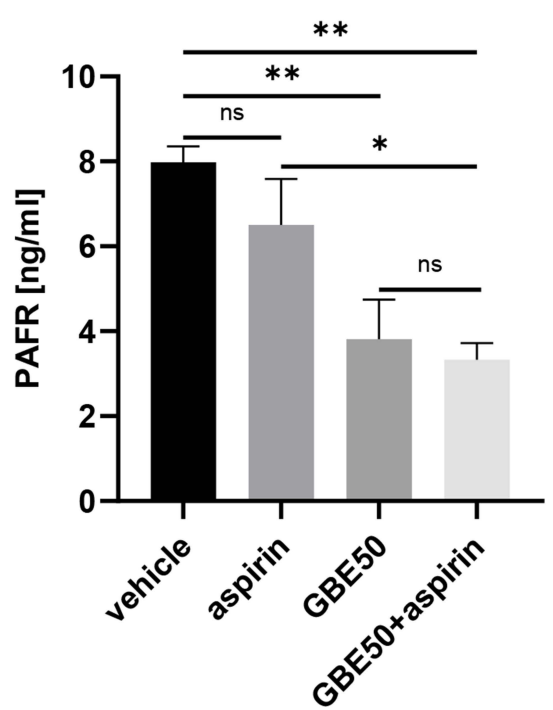

Figure 7 Effects of GBE50, aspirin and their combination on synthesis of CAMP and TXB2, and expression of PAFR.

Notes: The effects of GBE50 $0.3 \mathrm{mg} / \mathrm{mL}$, aspirin $0.3 \mathrm{mg} / \mathrm{mL}$ and $0.3 \mathrm{mg} / \mathrm{mL}$ GBE50 $+0.3 \mathrm{mg} / \mathrm{mL}$ aspirin on synthesis of cAMP (A), secretion of TXB2 (B) and expression with levels of PAFR $(\mathbf{C})$. Results were mean \pm S.E.M. of three independent experiments. ${ }^{*} p<0.05$, ${ }^{*} p<0.01$, $* * * p<0.00$ I.

Abbreviations: cAMP, cyclic adenosine monophosphate; TXB2, thromboxane B2; PAFR, platelet activating factor receptor; GBE50, Ginkgo biloba extract 50; ns, not significant. 
which did not differ significantly with effects obtained with vehicle alone. Instead, the levels of PAFR on platelet in drug combination group were significantly lower when compared with aspirin alone group, although there were no significant differences when compared with the GBE50 group.

\section{Discussion}

Our study indicated that the combination of GBE50 and aspirin displayed synergistic or additive antiplatelet effects. Other studies have also reported the efficacy of GBE50 in combination with antiplatelet drugs. Previous clinical studies have reported that EGb761 $(300 \mathrm{mg} / \mathrm{d})$ combined with aspirin does not enhance the antiplatelet effect in patients with peripheral artery disease. However, the results showed a decreased platelet aggregation in patients in the combination group under all inducers. It might be due to the insufficient sample size $(n=21$ placebo; $n=23$ ginkgo), there was no statistical difference in the results. In addition, no adverse bleeding events were observed in the combination group. ${ }^{34}$ Another clinical study of acute ischemic stroke also showed that there was no difference in the incidence of major bleeding events between GBE $(150 \mathrm{mg} / \mathrm{d})$ combined with aspirin group and aspirin monotherapy group. ${ }^{53}$ Cilostazol was also reported to exert stronger antiplatelet effect when combined with GBE in human platelets in vitro. ${ }^{31}$ Another clinical trial with small samples observed that GBE $(80 \mathrm{mg} / \mathrm{d})$ with cilostazol showed a remarkable, but not statistically significant, increase in inhibition of platelet aggregation. ${ }^{54}$ In addition, these studies all found that the combination did not prolong bleeding time and clotting time. Other clinical trial has also shown that the use of ticlopidine with GBE $(160 \mathrm{mg} / \mathrm{d})$ had a more significant effect on reducing platelet aggregation for secondary prevention of stroke. There was no increase in bleeding events. $^{33,55}$ These studies indicated that GBE combined with antiplatelet drugs appears to be more effective and does not cause higher risk of bleeding. However, most of these studies were small sample trials, a large cohort study with long-term follow-up might be needed to evaluate the possible pharmacodynamic interaction. In addition, the clinical dosage of GBE as a plant extract also needs to be evaluated as the dosages in reported studies are diverse. Animal experiments have shown that flavonol glycosides and aglycone conjugates could be substantially detected in plasma after dosing with ginkgo extracts, and the levels of systemic exposure to flavonols and terpene lactones were significant and increased in GBE50 dose-dependent manner. ${ }^{36,56}$ These might provide references for the clinical dosage of GBE50 to exert antiplatelet effect.

Previous studies evaluating drug combinations showed that the synergistic effects of drugs were mostly attributed to differences in the mechanisms of action. ${ }^{57,58}$ When drug targets overlap, this could lead to target competition, which could strongly influence their combination effects. In our in vitro platelet aggregation experiments, GBE50 and aspirin showed different inhibition intensities on platelet aggregation and combination treatment with the two drugs displayed prominent effects under all inducers of aggregation. The network pharmacology analysis also revealed that GBE50 and aspirin could interfere with platelet aggregation through different targets and pathways. There were only ten targets and one pathway overlapped for both GBE50 and aspirin, although the combination of the two drugs expanded the targets which could potentially regulate platelet aggregation. Our findings indicated that the combination would lead to synergistic antiplatelet effects. The synergy analysis by Synergyfinder2 showed that GBE50 and aspirin could exert synergistic or additive antiplatelet effects on platelet aggregation. Network pharmacology analysis showed consistently that both GBE50 and aspirin had different antiplatelet targets. GBE50 could influence platelet aggregation by targeting more pathways than aspirin, and thus, its combination with aspirin exerted a stronger inhibitory effect on platelet aggregation, which in turn, might positively enhance the antiplatelet effects of aspirin.

GO and KEGG enrichment analyses revealed that GBE50 could regulate platelet aggregation through a variety of pathways and biological processes. The degradation of cAMP is a common factor in the intracellular pathway of AA, PAF, ADP and collagen-induced platelet aggregation processes. Increased cAMP levels inhibit cytoskeletal rearrangement and fibrinogen receptor activation, resulting in a dull platelet response and inhibition platelet activation. ${ }^{9,59}$ Antiplatelet drugs acting on this pathway, such as cilostazol and dipyridamole, have been widely used in the clinic. ${ }^{5}$ Our data showed that GBE50 treatment combined with aspirin increased cAMP levels in platelets more significantly than drugs alone, they could ensure resting platelets maintain lower reactivity to stimulation.

The AA metabolic pathway is an important signaling pathway in platelet aggregation. When platelets are activated, AA is released from the plasma membrane of 
platelets and is converted to TXA2 by COX and thromboxane synthase. TXA2 transfers outside the cell to bind to the thromboxane receptor on the cell surface, which in turn stimulates platelet activation and recruits more platelets. The most representative antiplatelet drug that interferes with this pathway is aspirin. ${ }^{5,60}$ Our findings showed that aspirin displayed a strong inhibitory effect on TXB2 synthesis. The combined exposure to aspirin and GBE50 showed a more prominent effect than exposure to either single drug, and exhibited a synergistic antiplatelet effect. This might be due to their different effects on AA metabolism. Phospholipases A2 (PLA2) is a class of enzymes that can hydrolyze membrane phospholipids to AA. GBE could inhibit cytoplasmic PLA activity, which then inhibited the release of AA. ${ }^{61,62}$ When exogenous AA was added to platelets as an inducer, GBE50 was unable to inhibit exogenous AA metabolism. GBE50 could only inhibit endogenous AA generation caused by platelet activation; thus, it exerted a weak effect on platelet aggregation and TXB2 generation induced by AA. Aspirin inhibits AA metabolism by inhibiting COX-1 activity. ${ }^{16}$ Once combined, GBE50 could inhibit endogenous AA production and aspirin could inhibit both exogenous and endogenous AA metabolism; thus, they showed greater inhibitory effect on TXB2 generation and had a synergistic antiplatelet effect in AA-induced platelet aggregation. However, the mechanism of their synergistic antiplatelet action requires further investigation.

PAF can specifically bind to PAFR on the platelet surface and induce platelet activation and aggregation, which leads to promote $\mathrm{Ca}^{+}$influx. $^{63,64}$ Ginkgolides isolated from the Ginkgo biloba tree is a kind of natural PAFR antagonists. ${ }^{18}$ Our findings showed that GBE50 could also decrease the level of PAFR. Aspirin could not exert a similar inhibitory effect on PAFR, although the combination of GBE50 and aspirin displayed stronger antiplatelet effects in PAF-induced platelet aggregation. This may be attributed to the suppression of the activation of nuclear factor-kappa B (NF- $\kappa \mathrm{B})$ activation by aspirin, ${ }^{65}$ which has been proven to promote platelet activation and aggregation. ${ }^{66,67}$ Furthermore, PAFPAFR signaling can activate this pathway. ${ }^{18,68}$ Therefore, the additive antiplatelet effects of this combination may be due to the strong effect of GBE50 on PAFR and the downregulation of aspirin on NF- $\mathrm{kB}$.

Other studies also found that GBE could inhibited platelet aggregation by activating matrix metalloprotein (MMP)-9 and then inhibited COX-1 or TXA2 synthase in platelets. In addition, activated MMP-9 increased the formation of cAMP. ${ }^{69}$ Liu et $\mathrm{al}^{70}$ showed that the antiplatelet effects of GBE might associated with the inhibition of the phosphatidylinositol 3 kinase (PI3K)/protein kinase B (AKT) pathway. In addition, Go and KEGG enrichment analyses showed that GBE50 could also affect some inflammatory and oxidative stress processes including NFkappa B signaling pathway, leukocyte transendothelial migration, p53 signaling pathway, leukocyte migration, reactive oxygen species metabolic process and transmembrane receptor protein tyrosine kinase signaling pathway. Platelet aggregation is closely associated with inflammation and oxidative stress. For example, in atherosclerosis, macrophage and lipid oxidation mediated endothelial cell injury can cause platelet aggregation. ${ }^{71-74}$ GBE50 showed remarkable anti-inflammatory and antioxidant effects. ${ }^{75,76}$ Perhaps, the antiplatelet effect of GBE50 is determined by its strong antioxidant and anti-inflammatory effects.

This study has several limitations. First, the platelets used in the experiment came from rabbits, not patients. Further studies are required to evaluate the synergistic antiplatelet effect of GBE50 and aspirin in human, and it is best to give drugs in vivo. Second, the experimental results in each assay were obtained from 3 to 5 independent experiments, more repeated experiments are needed for further verification of antiplatelet effect of GBE50 and aspirin. Finally, we did not identify the most potent active ingredient in GBE50. The candidate components screened by TCMSP can be analyzed separately in the follow-up study to find the component or drug combination with the strongest synergistic antiplatelet effect.

\section{Conclusion}

Our study provided important evidence that GBE50 combined with aspirin could enhance the antiplatelet effects compared with monotherapy. These two drugs played synergistic or additive role in restraining platelet aggregation. The study highlighted the value and potential application of GBE50 as a supplementary therapy to treat thrombosis-related disease. Further studies are planned to assess the synergistic antiplatelet effect of GBE50 combined with aspirin and risk of bleeding in patients.

\section{Acknowledgments}

This work was supported by the National Key Research and Development Program of China (No. 2019YFC1711603, 2019YFC1708504) and the National Natural Science Foundation of China (No. 81771288). This work was also supported by Science and Technology Commission of 
Shanghai Municipal (No. 18401900600). SPH Xing Ling Sci. \& Tech. Pharmaceutical Co., Ltd. provided GBE50 for this study.

\section{Disclosure}

The authors report no conflicts of interest in this work.

\section{References}

1. McFadyen JD, Schaff M, Peter K. Current and future antiplatelet therapies: emphasis on preserving haemostasis. Nat Rev Cardiol. 2018;15(3):181-191. doi:10.1038/nrcardio.2017.206

2. Rengasamy KRR, Khan H, Ahmad I, et al. Bioactive peptides and proteins as alternative antiplatelet drugs. Med Res Rev. 2019;39 (6):2153-2171. doi:10.1002/med.21579

3. Benjamin EJ, Virani SS, Callaway CW, et al. Heart disease and stroke statistics-2018 update: a report from the American heart association. Circulation. 2018;137(12):e67-e492.

4. Estevez B, Du X. New concepts and mechanisms of platelet activation signaling. Physiology (Bethesda). 2017;32(2):162-177.

5. Xiang Q, Pang X, Liu Z, et al. Progress in the development of antiplatelet agents: focus on the targeted molecular pathway from bench to clinic. Pharmacol Ther. 2019;203:107393. doi:10.1016/j. pharmthera.2019.107393

6. van der Meijden PEJ, Heemskerk JWM. Platelet biology and functions: new concepts and clinical perspectives. Nat Rev Cardiol. 2019;16(3):166-179. doi:10.1038/s41569-018-0110-0

7. Yeung J, Li W, Holinstat M. Platelet signaling and disease: targeted therapy for thrombosis and other related diseases. Pharmacol Rev. 2018;70(3):526-548. doi:10.1124/pr.117.014530

8. Lordan R, Tsoupras A, Zabetakis I. Platelet activation and prothrombotic mediators at the nexus of inflammation and atherosclerosis: potential role of antiplatelet agents. Blood Rev. 2020;45:100694.

9. Knowles RB, Warner TD. Anti-platelet drugs and their necessary interaction with endothelial mediators and platelet cyclic nucleotides for therapeutic efficacy. Pharmacol Ther. 2019;193:83-90. doi:10.1016/j.pharmthera.2018.08.004

10. Zeng Y, Liu J, Zhang Q, et al. The traditional uses, phytochemistry and pharmacology of Sarcandra glabra (Thunb.) nakai, a Chinese herb with potential for development: review. Front Pharmacol. 2021;12:652926. doi:10.3389/fphar.2021.652926

11. Li XJ, Tang SQ, Huang H, et al. Acanthopanax henryi: review of botany, phytochemistry and pharmacology. Molecules. 2021;26(8):2215.

12. Chen M, Ding Y, Tong Z. Efficacy and safety of Sophora flavescens (Kushen) based traditional Chinese medicine in the treatment of ulcerative colitis: clinical evidence and potential mechanisms. Front Pharmacol. 2020;11:603476. doi:10.3389/fphar.2020.603476

13. Dai YJ, Wan SY, Gong SS, Liu JC, Li F, Kou JP. Recent advances of traditional Chinese medicine on the prevention and treatment of COVID-19. Chin J Nat Med. 2020;18(12):881-889.

14. Zhang L, Yu J, Zhou Y, Shen M, Sun L. Becoming a faithful defender: traditional Chinese medicine against coronavirus disease 2019 (COVID-19). Am J Chin Med. 2020;48(4):763-777. doi:10.1142/ S0192415X2050038X

15. Kumar A, Jaitak V. Natural products as multidrug resistance modulators in cancer. Eur J Med Chem. 2019;176:268-291. doi:10.1016/j. ejmech.2019.05.027

16. Fuster V, Sweeny JM. Aspirin: a historical and contemporary therapeutic overview. Circulation. 2011;123(7):768-778. doi:10.1161/ CIRCULATIONAHA.110.963843

17. Liu L, Wang Y, Zhang J, Wang S. Advances in the chemical constituents and chemical analysis of Ginkgo biloba leaf, extract, and phytopharmaceuticals. J Pharm Biomed Anal. 2021;193:113704. doi:10.1016/j.jpba.2020.113704
18. Lordan R, Tsoupras A, Zabetakis I, Demopoulos CA. Forty years since the structural elucidation of platelet-activating factor (PAF): historical, current, and future research perspectives. Molecules. 2019;24(23):4414. doi:10.3390/molecules24234414

19. Hyland IK, O'Toole RF, Smith JA, Bissember AC. Progress in the development of platelet-activating factor receptor (PAFr) antagonists and applications in the treatment of inflammatory diseases. ChemMedChem. 2018;13(18):1873-1884. doi:10.1002/ cmdc. 201800401

20. Koltai M, Hosford D, Guinot P, Esanu A, Braquet P. Platelet activating factor (PAF). A review of its effects, antagonists and possible future clinical implications (Part I). Drugs. 1991;42(1):9-29. doi:10.2165/00003495-199142010-00002

21. Constantinos A, Karantonis HC, Antonopoulou S. Platelet activating factor - a molecular link between atherosclerosis theories. Eur J Lipid Sci Technol. 2003;105(11):705-716. doi:10.1002/ejlt.200300845

22. Achete de Souza G, de Marqui SV, Matias JN, Guiguer EL, Barbalho SM. Effects of Ginkgo biloba on diseases related to oxidative stress. Planta Med. 2020;86(6):376-386. doi:10.1055/a-1109-3405

23. Singh SK, Srivastav S, Castellani RJ, Plascencia-Villa G, Perry G. Neuroprotective and antioxidant effect of ginkgo biloba extract against $\mathrm{AD}$ and other neurological disorders. Neurotherapeutics. 2019;16(3):666-674. doi:10.1007/s13311-019-00767-8

24. Kang JM, Lin S. Ginkgo biloba and its potential role in glaucoma. Curr Opin Ophthalmol. 2018;29(2):116-120. doi:10.1097/ ICU.0000000000000459

25. Zheng W, Xiang YQ, Ng CH, Ungvari GS, Chiu HF, Xiang YT. Extract of Ginkgo biloba for tardive dyskinesia: meta-analysis of randomized controlled trials. Pharmacopsychiatry. 2016;49 (3):107-111. doi:10.1055/s-0042-102884

26. Mahmoud F, Abul H, Onadeko B, Khadadah M, Haines D, Morgan G. In vitro effects of Ginkgolide B on lymphocyte activation in atopic asthma: comparison with cyclosporin A. Jpn J Pharmacol. 2000;83(3):241-245. doi:10.1016/S0021-5198(19)30590-6

27. Rui TQ, Zhang L, Qiao HZ, et al. Preparation and physicochemical and pharmacokinetic characterization of Ginkgo lactone nanosuspensions for antiplatelet aggregation. J Pharm Sci. 2016;105(1):242-249. doi:10.1016/j.xphs.2015.10.002

28. Kudolo GB, Dorsey S, Blodgett J. Effect of the ingestion of Ginkgo biloba extract on platelet aggregation and urinary prostanoid excretion in healthy and type 2 diabetic subjects. Thromb Res. 2002;108 (2-3):151-160. doi:10.1016/S0049-3848(02)00394-8

29. Dutta-Roy AK, Gordon MJ, Kelly C, et al. Inhibitory effect of Ginkgo biloba extract on human platelet aggregation. Platelets. 1999;10(5):298-305. doi:10.1080/09537109975933

30. Korth R, Nunez D, Bidault J, Benveniste J. Comparison of three paf-acether receptor antagonist ginkgolides. Eur $J$ Pharmacol. 1988;152(1-2):101-110. doi:10.1016/0014-2999(88)90840-0

31. Ryu KH, Han HY, Lee SY, et al. Ginkgo biloba extract enhances antiplatelet and antithrombotic effects of cilostazol without prolongation of bleeding time. Thromb Res. 2009;124(3):328-334. doi:10.1016/j.thromres.2009.02.010

32. Chan AL, Leung HW, Wu JW, Chien TW. Risk of hemorrhage associated with co-prescriptions for Ginkgo biloba and antiplatelet or anticoagulant drugs. J Altern Complement Med. 2011;17 (6):513-517. doi:10.1089/acm.2010.0295

33. Kellermann AJ, Kloft C. Is there a risk of bleeding associated with standardized Ginkgo biloba extract therapy? A systematic review and meta-analysis. Pharmacotherapy. 2011;31(5):490-502. doi:10.1592/ phco.31.5.490

34. Gardner CD, Zehnder JL, Rigby AJ, Nicholus JR, Farquhar JW. Effect of Ginkgo biloba (EGb 761) and aspirin on platelet aggregation and platelet function analysis among older adults at risk of cardiovascular disease: a randomized clinical trial. Blood Coagul Fibrinolysis. 2007;18 (8):787-793. doi:10.1097/MBC.0b013e3282f102b1 
35. Diamond BJ, Shiflett SC, Feiwel N, et al. Ginkgo biloba extract: mechanisms and clinical indications. Arch Phys Med Rehabil. 2000;81(5):668-678.

36. Li L, Zhao Y, Du F, et al. Intestinal absorption and presystemic elimination of various chemical constituents present in GBE50 extract, a standardized extract of Ginkgo biloba leaves. Curr Drug Metab. 2012;13(5):494-509. doi:10.2174/1389200211209050494

37. Clark JC, Kavanagh DM, Watson S, et al. Adenosine and forskolin inhibit platelet aggregation by collagen but not the proximal signalling events. Thromb Haemost. 2019;119(7):1124-1137. doi:10.1055/ s-0039-1688788

38. Chen P, Zhang D, Li M, et al. Discovery of novel, potent, isosteviol-based antithrombotic agents. Eur $J$ Med Chem. 2019;183:111722. doi:10.1016/j.ejmech.2019.111722

39. Wu QL, Dong J, Zeng HW, Lv C, Liu AJ, Zhang WD. Monitoring antiplatelet aggregation in vivo and in vitro by microtiter plate method. J Cardiovasc Pharmacol. 2020;75(4):314-320. doi:10.1097/FJC.0000000000000801

40. Chan MV, Warner TD. Standardised optical multichannel (optimul) platelet aggregometry using high-speed shaking and fixed time point readings. Platelets. 2012;23(5):404-408. doi:10.3109/ 09537104.2011.603066

41. Ianevski A, Giri AK, Aittokallio T. SynergyFinder 2.0: visual analytics of multi-drug combination synergies. Nucleic Acids Res. 2020;48 (W1):W488-w493. doi:10.1093/nar/gkaa216

42. Ru J, Li P, Wang J, et al. TCMSP: a database of systems pharmacology for drug discovery from herbal medicines. $J$ Cheminform. 2014;6:13. doi:10.1186/1758-2946-6-13

43. Xu X, Zhang W, Huang C, et al. A novel chemometric method for the prediction of human oral bioavailability. Int $J$ Mol Sci. 2012;13 (6):6964-6982. doi:10.3390/ijms13066964

44. Tao W, Xu X, Wang X, et al. Network pharmacology-based prediction of the active ingredients and potential targets of Chinese herbal Radix Curcumae formula for application to cardiovascular disease. J Ethnopharmacol. 2013;145(1):1-10. doi:10.1016/j. jep.2012.09.051

45. Daina A, Michielin O, Zoete V. SwissTargetPrediction: updated data and new features for efficient prediction of protein targets of small molecules. Nucleic Acids Res. 2019;47(W1):W357-w364 doi:10.1093/nar/gkz382

46. Wang L, Ma C, Wipf P, Liu H, Su W, Xie XQ. TargetHunter: an in silico target identification tool for predicting therapeutic potential of small organic molecules based on chemogenomic database. AAPS J. 2013;15(2):395-406. doi:10.1208/s12248-012-9449-Z

47. Wishart DS, Feunang YD, Guo AC, et al. DrugBank 5.0: a major update to the DrugBank database for 2018. Nucleic Acids Res. 2018;46(D1):D1074-d1082. doi:10.1093/nar/gkx1037

48. Wang JH, Zhao LF, Wang HF, et al. GenCLiP 3: mining human genes' functions and regulatory networks from PubMed based on cooccurrences and natural language processing. Bioinformatics. 2019:1973-1975. doi:10.1093/bioinformatics/btz807

49. Bardou P, Mariette J, Escudie F, Djemiel C, Klopp C. jvenn: an interactive Venn diagram viewer. BMC Bioinform. 2014;15(1):293. doi:10.1186/1471-2105-15-293

50. Shannon P, Markiel A, Ozier O, et al. Cytoscape: a software environment for integrated models of biomolecular interaction networks Genome Res. 2003;13(11):2498-2504. doi:10.1101/gr.1239303

51. Zhou Y, Zhou B, Pache L, et al. Metascape provides a biologist-oriented resource for the analysis of systems-level datasets. Nat Commun. 2019;10(1):1523. doi:10.1038/s41467-01909234-6

52. Bindea G, Mlecnik B, Hackl H, et al. ClueGO: a cytoscape plug-in to decipher functionally grouped gene ontology and pathway annotation networks. Bioinformatics. 2009;25(8):1091-1093. doi:10.1093/bioinformatics/btp101
53. Li S, Zhang X, Fang Q, et al. Ginkgo biloba extract improved cognitive and neurological functions of acute ischaemic stroke: a randomised controlled trial. Stroke Vasc Neurol. 2017;2 (4):189-197. doi:10.1136/svn-2017-000104

54. Kim HS, Kim GY, Yeo CW, et al. The effect of Ginkgo biloba extracts on the pharmacokinetics and pharmacodynamics of cilostazol and its active metabolites in healthy Korean subjects. $\mathrm{Br} J \mathrm{Clin}$ Pharmacol. 2014;77(5):821-830. doi:10.1111/bcp.12236

55. Hong JM, Shin DH, Lim YA, Lee JS, Joo IS. Ticlopidine with Ginkgo Biloba extract: a feasible combination for patients with acute cerebral ischemia. Thromb Res. 2013;131(4):e147-153. doi:10.1016/j.thromres.2013.01.026

56. Chen F, Li L, Xu F, et al. Systemic and cerebral exposure to and pharmacokinetics of flavonols and terpene lactones after dosing standardized Ginkgo biloba leaf extracts to rats via different routes of administration. Br J Pharmacol. 2013;170(2):440-457. doi:10.1111/ bph. 12285

57. Gkourogianni AV, Kiouptsi K, Koloka V, et al. Synergistic effect of peptide inhibitors derived from the extracellular and intracellular domain of $\alpha(\mathrm{IIb})$ subunit of integrin $\alpha(\mathrm{IIb}) \beta(3)$ on platelet activation and aggregation. Platelets. 2018;29(1):34-40. doi:10.1080/ 09537104.2017.1293804

58. Bouhtit F, Najar M, Moussa Agha D, et al. New anti-leukemic effect of carvacrol and thymol combination through synergistic induction of different cell death pathways. Molecules. 2021;26(2):2. doi:10.3390/ molecules 26020410

59. Smolenski A. Novel roles of cAMP/cGMP-dependent signaling in platelets. J Thromb Haemost. 2012;10(2):167-176. doi:10.1111/ j.1538-7836.2011.04576.x

60. Murphy PT, Grace S, Glavey S, Quinn J. Rivaroxaban may increase platelet activation in vivo via thromboxane A2. Circ Res. 2019;125 (2):e9. doi:10.1161/CIRCRESAHA.119.315453

61. Zhao Z, Liu N, Huang J, Lu PH, Xu XM. Inhibition of cPLA2 activation by Ginkgo biloba extract protects spinal cord neurons from glutamate excitotoxicity and oxidative stress-induced cell death. J Neurochem. 2011;116(6):1057-1065. doi:10.1111/j.14714159.2010.07160.x

62. Ong WY, Farooqui T, Kokotos G, Farooqui AA. Synthetic and natural inhibitors of phospholipases A2: their importance for understanding and treatment of neurological disorders. ACS Chem Neurosci. 2015;6(6):814-831. doi:10.1021/ acschemneuro.5b00073

63. Rasheed H, Saeed SA. Involvement of thromboxane A2 and tyrosine kinase in the synergistic interaction of platelet activating factor and calcium ionophore A23187 in human platelet aggregation. Exp Mol Med. 2004;36(3):220-225. doi:10.1038/emm.2004.30

64. Saeed SA, Rasheed H. Calcium-dependent synergistic interaction of platelet activating factor and epinephrine in human platelet aggregation. Acta Pharmacol Sin. 2003;24(1):31-36.

65. Kopp E, Ghosh S. Inhibition of NF-kappa B by sodium salicylate and aspirin. Science. 1994;265(5174):956-959. doi:10.1126/ science. 8052854

66. Hsia CW, Wu MP, Shen MY, Hsia CH, Chung CL, Sheu JR. Regulation of human platelet activation and prevention of arterial thrombosis in mice by auraptene through inhibition of NF-kappaB pathway. Int $J$ Mol Sci. 2020;21(13):4810. doi:10.3390/ ijms21134810

67. Satti HH, Khaleel EF, Badi RM, Elrefaie AO, Mostafa DG. Antiplatelet activity of astaxanthin in control- and high cholesterol-fed rats mediated by down-regulation of P2Y12, inhibition of NF-kappaB, and increasing intracellular levels of cAMP. Platelets. 2020;1-10. doi:10.1080/09537104.2020.1756237

68. Liu Y, Shields LBE, Gao Z, et al. Current understanding of platelet-activating factor signaling in central nervous system diseases. Mol Neurobiol. 2017;54(7):5563-5572. doi:10.1007/ s12035-016-0062-5 
69. Cho HJ, Nam KS. Inhibitory effect of ginkgolide B on platelet aggregation in a cAMP- and cGMP-dependent manner by activated MMP-9. J Biochem Mol Biol. 2007;40(5):678-683.

70. Liu X, Zhao G, Yan Y, Bao L, Chen B, Qi R. Ginkgolide B reduces atherogenesis and vascular inflammation in $\mathrm{ApoE}(-/-)$ mice. PLoS One. 2012;7(5):e36237. doi:10.1371/journal.pone.0036237

71. Libby P, Ridker PM, Hansson GK. Leducq Transatlantic Network on A. Inflammation in atherosclerosis: from pathophysiology to practice. $J$ Am Coll Cardiol. 2009;54(23):2129-2138. doi:10.1016/j. jacc.2009.09.009

72. Suarez-Rivero JM, Pastor-Maldonado CJ, Povea-Cabello S, et al. From mitochondria to atherosclerosis: the inflammation path. Biomedicines. 2021;9(3):258. doi:10.3390/biomedicines9030258

73. Malekmohammad K, Sewell RDE, Rafieian-Kopaei M. Antioxidants and atherosclerosis: mechanistic aspects. Biomolecules. 2019;9 (8):301. doi:10.3390/biom9080301
74. Summerhill V, Karagodin V, Grechko A, Myasoedova V, Orekhov A. Vasculoprotective role of olive oil compounds via modulation of oxidative stress in atherosclerosis. Front Cardiovasc Med. 2018;5:188. doi:10.3389/fcvm.2018.00188

75. Tian J, Liu Y, Chen K. Ginkgo biloba extract in vascular protection: molecular mechanisms and clinical applications. Curr Vasc Pharmacol. 2017;15(6):532-548. doi:10.2174/ 1570161115666170713095545

76. Diamond BJ, Bailey MR. Ginkgo biloba: indications, mechanisms, and safety. Psychiatr Clin North Am. 2013;36(1):73-83. doi:10.1016/ j.psc.2012.12.006

\section{Publish your work in this journal}

Drug Design, Development and Therapy is an international, peerreviewed open-access journal that spans the spectrum of drug design and development through to clinical applications. Clinical outcomes, patient safety, and programs for the development and effective, safe, and sustained use of medicines are a feature of the journal, which has also been accepted for indexing on PubMed Central. The manuscript management system is completely online and includes a very quick and fair peer-review system, which is all easy to use. Visit http://www. dovepress.com/testimonials.php to read real quotes from published authors. 